\title{
The Weak Galerkin Method for Elliptic Eigenvalue Problems
}

\author{
Qilong Zhai ${ }^{1}$, Hehu Xie ${ }^{2,3}$, Ran Zhang ${ }^{1, *}$ and Zhimin Zhang ${ }^{4,5}$ \\ ${ }^{1}$ Department of Mathematics, Jilin University, Changchun 130012, China. \\ 2 LSEC, ICMSEC, Academy of Mathematics and Systems Science, Chinese Academy \\ of Sciences, Beijing 100190, P.R. China. \\ 3 School of Mathematical Sciences, University of Chinese Academy of Sciences, \\ Beijing 100049, P.R. China. \\ 4 Beijing Computational Science Research Center, Beijing, 100193, China. \\ ${ }^{5}$ Department of Mathematics, Wayne State University, Detroit, MI 48202, USA.
}

Received 8 August 2018; Accepted (in revised version) 31 August 2018

\begin{abstract}
This article is devoted to studying the application of the weak Galerkin (WG) finite element method to the elliptic eigenvalue problem with an emphasis on obtaining lower bounds. The WG method uses discontinuous polynomials on polygonal or polyhedral finite element partitions. The non-conforming finite element space of the WG method is the key of the lower bound property. It also makes the WG method more robust and flexible in solving eigenvalue problems. We demonstrate that the WG method can achieve arbitrary high convergence order. This is in contrast with existing nonconforming finite element methods which can provide lower bound approximations by linear finite elements. Numerical results are presented to demonstrate the efficiency and accuracy of the theoretical results.
\end{abstract}

AMS subject classifications: 65N30, 65N15, 65N12, 74N20, 35B45, 35J50, 35J35

Key words: Weak Galerkin finite element method, elliptic eigenvalue problem, lower bounds, error estimate.

\section{Introduction}

The eigenvalue problems of partial differential equations arising from the scientific research and engineering have received more and more attentions recently $[6,9,14,28]$. Among the PDE eigenvalue problems, the elliptic eigenvalues are closely related to Poincaré constant in the Sobolev theory [19,34], and play an important role in the spectral distribution of nonlinear equations [33]. In physical applications, the eigenvalues

*Corresponding author. Email addresses: diql15@mails.jlu.edu.cn (Q. Zhai), hhxie@lsec.cc.ac.cn (H. Xie), zhangran@jlu.edu.cn (R. Zhang), zmzhang@csrc.ac.cn, zzhang@math.wayne.edu (Z.Zhang) 
often have close relationship with vibrations, especially the sympathetic vibration phenomenon. Most elastic bodies vibrate at certain frequency and respond to external vibrations. Details of these applications can be found in $[7,15,20]$. In addition to the above mentioned applications, the elliptic type eigenvalue problems are also useful in many other areas, such as plasma physics in fusion experiments and astrophysics, the petroleum reservoir simulation, the linear stability of flows in fluid mechanics, and electronic band structure calculations, etc. (see $[1,4,14,21]$ and references therein).

There have been numerous efforts in finding numerical solutions of elliptic eigenvalue problems. The finite element method is the most studied method (see, e.g., $[3,4,6,16,17])$. Due to the Rayleigh quotient and the minimum-maximum principle, any standard conforming finite element method $[13,35]$ can only provide upper bounds for the eigenvalues. However, when the eigenvalues are all real numbers, it is desirable to obtain both upper bounds and lower bounds [27].

There are mainly two approaches to find lower bounds of eigenvalues. The first approach is a post-processing procedure $[22,26]$. The main drawback of this approach is that an auxiliary problem must be solved and the order of convergence is reduced as a result. The second approach is the construction of special nonconforming finite elements to obtain lower bounds. In [23], three types of nonconforming elements by the finite element error expansion technique were studied to provide lower bounds of the eigenvalues. We refer interested readers to $[5,25]$ for additional studies on other nonconforming finite element approaches in obtaining lower bounds for eigenvalues. There are mainly two difficulties in the numerical approximation of eigenvalue problems with nonconforming FEM. One is the construction of high order finite element spaces which give high order numerical solutions. The other difficulty is the construction of finite element spaces for three dimensional problems.

The goal of this paper is to overcome the aforementioned difficulties using the weak Galerkin method. The WG method was first proposed in [37], and further developed in $[8,29,31,36,38,44,45,47,48]$. Recently, the weak Galerkin method has been extended to elliptic interface problems [10], linear hyperbolic equations [43], Navier-Stokes equations [46, 49], Helmholtz equations [11,32], and discrete maximum principles [18,39]. In the WG method, differential operators are approximated by weak forms as distributions over a set of generalized functions. It has been demonstrated that the WG method is highly flexible and robust as a numerical technique employing discontinuous piecewise polynomials on polygonal or polyhedral finite element partitions. As a class of nonconforming finite element method, the finite element space of WG, for the same degree of polynomial, is larger than that of the standard finite element methods, which makes it possible to obtain lower bounds due to the Rayleigh quotient. Comparing with other nonconforming finite element for eigenvalue problems, our approach of solving elliptic eigenvalue problems with the WG method has the following advantages, which constitute the main contributions of this paper.

- Our method is capable of obtaining lower bounds with higher order of accuracy. 
In the WG method, high order piecewise polynomial finite element spaces are constructed, and the high order convergence can be reached as a result.

- Unlike other non-conforming methods, the WG method for solving 2D eigenvalue problems can be seamlessly extended to 3D cases.

- Polytopal meshes can be adopted in the WG method. Since the WG finite element space consists of discontinuous piecewise polynomials, it can easily handle arbitrary polytopal meshes, including hanging nodes. In comparison, other nonconforming finite methods are mostly restricted to triangular or quadrilateral meshes.

The challenges for solving the lower bound problems by the WG method arise from two aspects. One aspect is that the WG finite element space is not a subspace of $H_{0}^{m}$ space or $L^{2}$ space, hence the classical analysis for conforming finite element method or nonconforming finite element method does not apply to the WG method directly. To overcome this difficulty, we first propose a general framework based on the expansion equality in Section 2, and apply this frame work to the WG method. The other aspect is that the lower bounds can not be obtained by simply rewriting the WG schemes in [37] or [48] for the eigenvalue problems. In Section 3 and Section 4, we obtain the asymptotic lower bound estimations by introducing a infinitesimal factor in the magnitude of $\mathcal{O}\left(h^{\varepsilon}\right)$ or $1 / \log (h)$ in the stabilization term. This factor will play a key role in obtaining the lower bounds.

An outline of the paper is as follows. In Section 2, we develop a framework for the application of WG method to eigenvalue problems. In Section 3, we apply the abstract framework to two types of elliptic eigenvalue problems: the Laplacian and biharmonic eigenvalue problems. In Section 4, we conduct several numerical experiments to demonstrate the efficiency of our methods. A few concluding remarks are given in Section 5.

\section{An abstract framework}

Suppose $\left(V,(\cdot, \cdot)_{V}\right)$ is a Hilbert space, $V_{c}$ and $V_{h}$ are two subspaces of $V$. In the WG method, $V_{c}$ usually denotes $H_{0}^{m}(\Omega)$, and $V_{h}$ is the corresponding WG finite element space. Let $a(\cdot, \cdot)$ be a bilinear form on $V_{c} \times V_{c}, a_{w}(\cdot, \cdot)$ be a bilinear form on $V_{h} \times V_{h}$, and $b(\cdot, \cdot)$ be a bilinear form on $V \times V$. We consider the eigenvalue problems:

Find $(\lambda, u) \in \mathbb{R} \times V_{c}$ and $\left(\lambda_{h}, u_{h}\right) \in \mathbb{R} \times V_{h}$ such that $b(u, u)=b\left(u_{h}, u_{h}\right)=1$ and

$$
\begin{aligned}
& a(u, v)=\lambda b(u, v), \quad \forall v \in V_{c}, \\
& a_{w}\left(u_{h}, v_{h}\right)=\lambda_{h} b\left(u_{h}, v_{h}\right), \quad \forall v_{h} \in V_{h} .
\end{aligned}
$$

We assume that the bilinear forms $a, a_{w}$ and $b$ have the following property:

Assumption (A1). $a, a_{w}$, and $b$ are symmetric, and for any $v \in V_{c}$ and $v_{h} \in V_{h}$,

$$
\begin{aligned}
& a(v, v) \geq \gamma_{c}\|v\|_{V}^{2}, \\
& a_{w}\left(v_{h}, v_{h}\right) \geq \gamma(h)\left\|v_{h}\right\|_{V}^{2},
\end{aligned}
$$


where $\gamma_{c}$ and $\gamma(h)$ are positive constants.

The eigenvalue problems (2.1) and (2.2) can be viewed as operator spectrum problems. Define two operators $K: V_{c} \rightarrow V_{c}$ and $K_{h}: V_{h} \rightarrow V_{h}$ satisfying

$$
\begin{aligned}
& a(K f, v)=b(f, v), \quad \forall v \in V_{c}, \\
& a_{w}\left(K_{h} f_{h}, v_{h}\right)=b\left(f_{h}, v_{h}\right), \quad \forall v_{h} \in V_{h} .
\end{aligned}
$$

Under Assumption (A1), $K$ and $K_{h}$ are well-defined. It is clear that $K$ and $K_{h}$ share the same eigensystem with (2.1)-(2.2).

Suppose $\mu$ is a nonzero eigenvalue of $K$ and $u$ is an eigenfunction corresponding to $\mu$, then we have

$$
K u=\mu u,
$$

i.e.

$$
a(u, v)=\frac{1}{\mu} a(K u, v)=\frac{1}{\mu} b(u, v), \quad \forall v \in V_{c} .
$$

Hence, $\left(\frac{1}{\mu}, u\right)$ is a solution of (2.1). Similarly, if $(\lambda, u)$ is solution of $(2.1)$, then $\left(\frac{1}{\lambda}, u\right)$ is an eigenpair of $K$. Thus, solving eigenvalue problems (2.1)-(2.2) is equivalent to finding the non-zero spectrum and corresponding eigenfunctions of $K$ and $K_{h}$.

We denote by $\sigma(K)$ the spectrum of $K$, and by $\rho(K)$ the resolvent set. $R_{z}(K)=(z I-$ $K)^{-1}$ represents the resolvent operator for any $z \in \rho(K)$. Let $\mu$ be a nonzero eigenvalue of $K$ with algebraic or geometric multiplicities $m$. Let $\Gamma_{\mu}$ be a circle in the complex plane centered at $\mu$ which lies in $\rho(K)$ and encloses no other points of $\sigma(K)$. The corresponding spectral projection is

$$
E_{\mu}(K)=\frac{1}{2 \pi \mathrm{i}} \int_{\Gamma_{\mu}} R_{z}(K) d z
$$

It is known that the range $R\left(E_{\mu}(K)\right)$ of $E_{\mu}(K)$ is the eigenspace corresponding to the eigenvalue $\mu$.

Denote by $\Pi_{c}: V \rightarrow V_{c}$ the orthogonal projection operator under $(\cdot, \cdot)_{V}$ inner-product. Then $K \Pi_{c}$ can be viewed as an operator from $V$ to $V$.

Lemma 2.1. Under Assumption (A1), the following identities hold

$$
\sigma(K)=\sigma\left(K \Pi_{c}\right) \backslash\{0\} \text {, and } R\left(E_{\mu}(K)\right)=R\left(E_{\mu}\left(K \Pi_{c}\right)\right), \quad \forall \mu \in \sigma(K) .
$$

Proof. Suppose $\mu \in \sigma(K)$ and $u \in R\left(E_{\mu}(K)\right)$. It follows from Assumption (A1) that $\mu>0$. Then we have

$$
K \Pi_{c} u=K u=\mu u,
$$


i.e. $\mu \in \sigma\left(K \Pi_{c}\right) \backslash\{0\}$ and $u \in R\left(E_{\mu}\left(K \Pi_{c}\right)\right)$.

On the other hand, if $\mu \in \sigma\left(K \Pi_{c}\right) \backslash\{0\}$ and $u \in R\left(E_{\mu}\left(K \Pi_{c}\right)\right)$, then $K u \in V_{c}$, and it follows that $u=\frac{1}{\mu} K u \in V_{c}$. Thus we have

$$
K u=K \Pi_{c} u=\mu u,
$$

i.e. $\mu \in \sigma(K)$ and $u \in R\left(E_{\mu}(K)\right)$. Thus the proof is completed.

Denote by $\Pi_{h}: V \rightarrow V_{h}$ the orthogonal projection operator under $(\cdot, \cdot)_{V}$ inner-product. Then $K_{h} \Pi_{h}$ can also be viewed as a operator from $V$ to $V$. Similar to Lemma 2.1, the following identities hold.

Lemma 2.2. Under Assumption (A1), there holds that

$$
\sigma\left(K_{h}\right)=\sigma\left(K_{h} \Pi_{h}\right) \backslash\{0\} \text {, and } R\left(E_{\mu_{h}}\left(K_{h}\right)\right)=R\left(E_{\mu_{h}}\left(K_{h} \Pi_{h}\right)\right), \quad \forall \mu_{h} \in \sigma\left(K_{h}\right) .
$$

From Lemmas 2.1-2.2, we conclude that solving eigenvalue problems (2.1)-(2.2) is equivalent to finding the non-zero spectrum and corresponding eigenfunctions of $K \Pi_{c}$ and $K_{h} \Pi_{h}$, respectively.

In order to get the final error estimates, we need $K \Pi_{c}$ and $K_{h} \Pi_{h}$ to be self-adjoint and compact. To this end, we make the following assumption.

Assumption (A2). $K$ and $K_{h}$ are compact.

Under this assumption, we have the following lemma.

Lemma 2.3. Under Assumptions (A1)-(A2), $K \Pi_{c}$ and $K_{h} \Pi_{h}$ are self-adjoint and compact.

Proof. For any $u, v \in V$, it follows from Assumption (A1) that

$$
\begin{aligned}
& \left(K \Pi_{c} u, v\right)_{V}=\left(K \Pi_{c} u, \Pi_{c} v\right)_{V}=b\left(\Pi_{c} u, \Pi_{c} v\right)=\left(\Pi_{c} u, K \Pi_{c} v\right)_{V}=\left(u, K \Pi_{c} v\right)_{V}, \\
& \left(K \Pi_{h} u, v\right)_{V}=\left(K \Pi_{h} u, \Pi_{h} v\right)_{V}=b\left(\Pi_{h} u, \Pi_{h} v\right)=\left(\Pi_{h} u, K \Pi_{h} v\right)_{V}=\left(u, K \Pi_{h} v\right)_{V} .
\end{aligned}
$$

Thus, $K \Pi_{c}$ and $K \Pi_{h}$ are self-adjoint.

Since $\Pi_{c}$ and $\Pi_{h}$ are bounded and $K$, and $K_{h}$ are compact, we have $K \Pi_{c}$ and $K_{h} \Pi_{h}$ are compact, which completes the proof.

In order to estimate the error of eigenvalues and eigenfunctions in (2.1)-(2.2), we need to estimate the approximation error $\left\|K \Pi_{c}-K_{h} \Pi_{h}\right\|_{V}$. However, the projection operator $\Pi_{h}$ is usually not explicitly defined in the numerical scheme. Hence, for the convenience of the analysis in the rest of the paper, we estimate $\left\|K \Pi_{c}-K_{h} \Pi_{h}\right\|_{V}$ in an indirect way. Suppose the following assumption holds.

Assumption (A3). There exists a bounded linear operator $Q_{h}: V \rightarrow V_{h}$ satisfying

$$
\begin{aligned}
& Q_{h} v_{h}=v_{h}, \quad \forall v_{h} \in V_{h}, \\
& b\left(Q_{h} w, v_{h}\right)=b\left(w, v_{h}\right), \quad \forall w \in V, v_{h} \in V_{h} .
\end{aligned}
$$


For any $\mu \in \sigma(K)$, since $R\left(E_{\mu}(K)\right) \subset V$, we have $\left.K \Pi_{\mathcal{c}}\right|_{R\left(E_{\mu}(K)\right)}=\left.K\right|_{R\left(E_{\mu}(K)\right)}$. Denote

$$
e_{h, \mu}=\left\|\left.\left(K-K_{h} Q_{h}\right)\right|_{R\left(E_{\mu}(K)\right)}\right\|_{V}
$$

and

$$
\delta_{h, \mu}=\sup _{\substack{u \in R\left(E_{\mu}(K)\right),\|u\|_{V}=1}}\left\|u-Q_{h} u\right\|_{V} .
$$

Then we have the following estimate.

Lemma 2.4. Under Assumptions (A1) and (A3), for any $\mu \in \sigma(K)$ we have

$$
\left\|\left.\left(K \Pi_{c}-K_{h} \Pi_{h}\right)\right|_{R\left(E_{\mu}(K)\right)}\right\|_{V} \leq e_{h, \mu}+\delta_{h, \mu} \gamma(h)^{-1} .
$$

Proof. For any $f \in R\left(E_{\mu}(K)\right)$, it follows from the definition of $K_{h}$ that

$$
a_{w}\left(K_{h}\left(Q_{h}-\Pi_{h}\right) f, v_{h}\right)=b\left(\left(Q_{h}-\Pi_{h}\right) f, v_{h}\right), \quad \forall v_{h} \in V_{h} .
$$

By taking $v_{h}=K_{h}\left(Q_{h}-\Pi_{h}\right) f$ and using Assumption (A1) we have

$$
\begin{aligned}
\gamma(h)\left\|K_{h}\left(Q_{h}-\Pi_{h}\right) f\right\|_{V}^{2} & \leq a_{w}\left(K_{h}\left(Q_{h}-\Pi_{h}\right) f, K_{h}\left(Q_{h}-\Pi_{h}\right) f\right) \\
& =b\left(\left(Q_{h}-\Pi_{h}\right) f, K_{h}\left(Q_{h}-\Pi_{h}\right) f\right) \\
& =b\left(f-\Pi_{h} f, K_{h}\left(Q_{h}-\Pi_{h}\right) f\right) \\
& \leq\left\|f-\Pi_{h} f\right\|_{V}\left\|K_{h}\left(Q_{h}-\Pi_{h}\right) f\right\|_{V} \\
& \leq \delta_{h, \mu}\left\|K_{h}\left(Q_{h}-\Pi_{h}\right) f\right\|_{V}
\end{aligned}
$$

which implies that

$$
\left\|K_{h}\left(Q_{h}-\Pi_{h}\right) f\right\|_{V} \leq \delta_{h, \mu} \gamma(h)^{-1} .
$$

From the triangle inequality we have

$$
\begin{aligned}
& \left\|\left.\left(K \Pi_{c}-K_{h} \Pi_{h}\right)\right|_{R\left(E_{\mu}(K)\right)}\right\|_{V} \\
\leq & \left\|\left.\left(K-K_{h} Q_{h}\right)\right|_{R\left(E_{\mu}(K)\right)}\right\|_{V}+\left\|\left.\left(K_{h} Q_{h}-K_{h} \Pi_{h}\right)\right|_{R\left(E_{\mu}(K)\right)}\right\|_{V} \\
\leq & e_{h, \mu}+\delta_{h, \mu} \gamma(h)^{-1},
\end{aligned}
$$

which completes the proof.

Suppose the errors $e_{h, \mu}$ and $\delta_{h, \mu}$ satisfy the following assumption.

Assumption (A4). $e_{h, \mu} \rightarrow 0$ as $h \rightarrow 0$, and $\delta_{h, \mu} \gamma(h)^{-1} \rightarrow 0$ as $h \rightarrow 0$.

From Lemma 2.4, we have the following estimate immediately. 
Lemma 2.5. Under Assumptions (A1)-(A4), for any $\mu \in \sigma(K)$ we have

$$
\left\|\left.\left(K \Pi_{c}-K_{h} \Pi_{h}\right)\right|_{R\left(E_{\mu}(K)\right)}\right\|_{V} \rightarrow 0 \text { as } h \rightarrow 0 .
$$

For a Banach space $H$ and its closed subspaces $M$ and $N$, define the distances as follows that

$$
\begin{aligned}
& \rho_{H}(x, N)=\inf _{y \in N}\|x-y\|_{H}, \quad \rho_{H}(M, N)=\sup _{\substack{x \in M,\|x\|_{H}=1}} \rho(x, N), \\
& \hat{\rho}_{H}(M, N)=\max \left\{\rho_{H}(M, N), \rho_{H}(N, M)\right\} .
\end{aligned}
$$

According to the Babŭska-Osborn's theory, we have the following estimation on eigenfunctions. The proof can be found in [3, Theorem 7.1].

Theorem 2.1. Under Assumptions (A1)-(A4), for any $\mu \in \sigma(K)$ we have

$$
\hat{\rho}_{V}\left(R\left(E_{\mu}(K)\right), R\left(E_{\mu, h}\left(K_{h}\right)\right) \leq C\left(e_{h, \mu}+\delta_{h} \gamma(h)^{-1}\right),\right.
$$

where the constants only depends on the eigenvalues of $K$.

Suppose $X$ is a Hilbert space equipped with inner-product $b(\cdot, \cdot)$, and $\|\cdot\|_{X}$ is the corresponding norm. In the elliptic eigenvalue problems, $X$ is $L^{2}(\Omega)$. Suppose $\Pi_{0}$ is the orthogonal projection from $V$ onto $X$ under $b(\cdot, \cdot)$. Then $\left.\Pi_{0} K\right|_{\Pi_{0} V_{c}}: \Pi_{0} V_{c} \rightarrow \Pi_{0} V_{c}$ and $\left.\Pi_{0} K_{h}\right|_{\Pi_{0} V_{h}}: \Pi_{0} V_{h} \rightarrow \Pi_{0} V_{h}$ are bounded linear operators.

Lemma 2.6. The following identities hold

$$
\sigma\left(\Pi_{0} K\right)=\sigma(K), \quad \sigma\left(\Pi_{0} K_{h}\right)=\sigma\left(K_{h}\right) .
$$

For each $\mu \in \sigma(K)$ and $\mu_{h} \in \sigma\left(K_{h}\right)$

$$
R\left(E_{\mu}\left(\Pi_{0} K\right)\right)=\Pi_{0} R\left(E_{\mu}(K)\right), \quad R\left(E_{\mu_{h}}\left(\Pi_{0} K_{h}\right)\right)=\Pi_{0} R\left(E_{\mu_{h}}\left(K_{h}\right)\right) .
$$

Proof. Suppose $(\mu, u)$ is an eigenpair of $K$, i.e.,

$$
K u=\mu u .
$$

Notice that from the definition of $K$, for any $v \in V_{c}$ we have

$$
a(K u, v)=b(u, v)=b\left(\Pi_{0} u, v\right)=a\left(K \Pi_{0} u, v\right),
$$

which implies that

$$
\mu u=K u=K \Pi_{0} u .
$$

By multiplying $\Pi_{0}$ on both side we have

$$
\mu \Pi_{0} u=\Pi_{0} K \Pi_{0} u .
$$


Thus, $\left(\mu, \Pi_{0} u\right)$ is an eigenpair of $\Pi_{0} K$, and then $\Pi_{0} R\left(E_{\mu}(K)\right) \subset R\left(E_{\mu}\left(\Pi_{0} K\right)\right)$.

Suppose $(\mu, \hat{u})$ is an eigenpair of $\Pi_{0} K$, i.e.,

$$
\Pi_{0} K \hat{u}=\mu \hat{u} .
$$

Denote $u=K \hat{u} \in V_{c}$, then for any $v \in V_{c}$ we have

$$
a(K u, v)=a(K(K \hat{u}), v)=b(K \hat{u}, v)=b\left(\Pi_{0} K \hat{u}, v\right)=\mu b(\hat{u}, v)=\mu a(K \hat{u}, v)=\mu a(u, v)
$$

which implies that

$$
K u=\mu u
$$

Notice that

$$
\Pi_{0} u=\Pi_{0} K \hat{u}=\mu \hat{u} .
$$

Hence, $(\lambda, u)$ is an eigenpair of $K$ with $\Pi_{0} u \in R\left(E_{\mu}\left(\Pi_{0} K\right)\right)$, and then $R\left(E_{\mu}\left(\Pi_{0} K\right)\right) \subset$ $\Pi_{0} R\left(E_{\mu}(K)\right)$. Thus, the proof is completed.

Denote $e_{h, \mu}^{\prime}=\left\|\left.\left(\Pi_{0} K-\Pi_{0} K_{h} Q_{h}\right)\right|_{R\left(E_{\mu}(K)\right)}\right\|_{X}$ and $\delta_{h}^{\prime}=\rho_{X}\left(V, V_{h}\right)$ which is defined in (2.6)(2.7). Suppose the errors $e_{h, \mu}^{\prime}$ and $\delta_{h}^{\prime}$ satisfy the following assumption.

Assumption (A5). $e_{h, \mu}^{\prime} \rightarrow 0$ as $h \rightarrow 0$, and $\delta_{h}^{\prime} \gamma(h)^{-1} \rightarrow 0$ as $h \rightarrow 0$.

Repeating the proof of Theorem 2.1, we have the following estimate.

Lemma 2.7. Under Assumptions (A1)-(A5), for any $\mu \in \sigma(K)$ we have

$$
\hat{\rho}_{X}\left(R\left(E_{\mu}\left(\Pi_{0} K\right)\right), R\left(E_{\mu, h}\left(\Pi_{0} K_{h}\right)\right) \leq C\left(e_{h, \mu}^{\prime}+\delta_{h}^{\prime} \gamma(h)^{-1}\right) .\right.
$$

Notice that for any $v \in V,\|v\|_{X}=\left\|\Pi_{0} v\right\|_{X}$. Then Lemma 2.7 can be written in the following form.

Theorem 2.2. Under Assumptions (A1)-(A5), for any $\mu \in \sigma(K)$ we have

$$
\hat{\rho}_{X}\left(R\left(E_{\mu}(K)\right), R\left(E_{\mu, h}\left(K_{h}\right)\right) \leq C\left(e_{h, \mu}^{\prime}+\delta_{h}^{\prime} \gamma(h)^{-1}\right) .\right.
$$

Now we turn to the estimation of the eigenvalues. The proof is based on an expansion formula, the idea is similar to Lemma 2.2 in [2].

Lemma 2.8. Under Assumption (A1), suppose $(\lambda, u)$ is the solution of (2.1) and $\left(\lambda_{h}, u_{h}\right)$ is the solution of (2.2). Then for any $v_{h} \in V_{h}$ we have the following expansion

$$
\begin{aligned}
\lambda-\lambda_{h}= & a(u, u)-a_{w}\left(v_{h}, v_{h}\right)+a_{w}\left(u_{h}-v_{h}, u_{h}-v_{h}\right) \\
& -\lambda_{h} b\left(u_{h}-v_{h}, u_{h}-v_{h}\right)-\lambda_{h}\left(b(u, u)-b\left(v_{h}, v_{h}\right)\right) .
\end{aligned}
$$


Proof. From (2.1)-(2.2), for any $v_{h} \in V_{h}$ we have

$$
\begin{aligned}
& \lambda_{h} b\left(u_{h}-v_{h}, u_{h}-v_{h}\right)+\lambda_{h}\left(b(u, u)-b\left(v_{h}, v_{h}\right)\right) \\
= & \lambda_{h} b\left(u_{h}-v_{h}, u_{h}-v_{h}\right)+\lambda_{h}\left(b\left(u_{h}, u_{h}\right)-b\left(v_{h}, v_{h}\right)\right) \\
= & 2 \lambda_{h} b\left(u_{h}, u_{h}\right)-2 \lambda_{h} b\left(u_{h}, v_{h}\right) \\
= & 2 \lambda_{h} b\left(u_{h}, u_{h}\right)-2 \lambda_{h} a_{w}\left(u_{h}, v_{h}\right) .
\end{aligned}
$$

Then, we obtain

$$
\begin{aligned}
\lambda-\lambda_{h}= & \lambda b(u, u)+\lambda b\left(u_{h}, u_{h}\right)-2 \lambda_{h} b\left(u_{h}, u_{h}\right) \\
= & a(u, u)+a_{w}\left(u_{h}, u_{h}\right)-2 \lambda_{h} a_{w}\left(u_{h}, v_{h}\right) \\
& -\lambda_{h} b\left(u_{h}-v_{h}, u_{h}-v_{h}\right)-\lambda_{h}\left(b(u, u)-b\left(v_{h}, v_{h}\right)\right) \\
= & a(u, u)-a_{w}\left(v_{h}, v_{h}\right)+a_{w}\left(u_{h}-v_{h}, u_{h}-v_{h}\right) \\
& -\lambda_{h} b\left(u_{h}-v_{h}, u_{h}-v_{h}\right)-\lambda_{h}\left(b(u, u)-b\left(v_{h}, v_{h}\right)\right),
\end{aligned}
$$

which completes the proof.

By substituting $v_{h}=Q_{h} u$ in Lemma 2.8, we have the following lemma immediately.

Lemma 2.9. Under Assumptions (A1) and (A3), suppose $(\lambda, u)$ is the solution of (2.1) and $\left(\lambda_{h}, u_{h}\right)$ is the solution of (2.2). Then for any $v_{h} \in V_{h}$ we have the following expansion

$$
\begin{aligned}
\lambda-\lambda_{h}= & a(u, u)-a_{w}\left(Q_{h} u, Q_{h} u\right)+a_{w}\left(u_{h}-Q_{h} u, u_{h}-Q_{h} u\right) \\
& -\lambda_{h} b\left(u-u_{h}, u-u_{h}\right) .
\end{aligned}
$$

Proof. It follows from the properties of $Q_{h} u$ in (A3) that

$$
\begin{aligned}
b(u, u)-b\left(Q_{h} u, Q_{h} u\right) & =b\left(u+Q_{h} u, u-Q_{h} u\right) \\
& =b\left(u-Q_{h} u, u-Q_{h} u\right) .
\end{aligned}
$$

Then by taking $v_{h}=Q_{h} u$ in Lemma 2.8 we have

$$
\begin{aligned}
\lambda-\lambda_{h}= & a(u, u)-a_{w}\left(Q_{h} u, Q_{h} u\right)+a_{w}\left(u_{h}-Q_{h} u, u_{h}-Q_{h} u\right) \\
& -\lambda_{h} b\left(u_{h}-Q_{h} u, u_{h}-Q_{h} u\right)-\lambda_{h}\left(b(u, u)-b\left(Q_{h} u, Q_{h} u\right)\right) \\
= & a(u, u)-a_{w}\left(Q_{h} u, Q_{h} u\right)+a_{w}\left(u_{h}-Q_{h} u, u_{h}-Q_{h} u\right)-\lambda_{h} b\left(u_{h}-Q_{h} u, u_{h}-Q_{h} u\right) \\
& -\lambda_{h} b\left(u-Q_{h} u, u-Q_{h} u\right)-2 \lambda_{h} b\left(u-Q_{h} u, u_{h}-Q_{h} u\right) \\
= & a(u, u)-a_{w}\left(Q_{h} u, Q_{h} u\right)+a_{w}\left(u_{h}-Q_{h} u, u_{h}-Q_{h} u\right) \\
& -\lambda_{h} b\left(u-u_{h}, u-u_{h}\right) .
\end{aligned}
$$

Thus the proof is completed.

Denote $\varepsilon_{h, u}=a(u, u)-a_{w}\left(Q_{h} u, Q_{h} u\right)$. Suppose the following assumption holds.

Assumption (A6). For any $\mu \in \sigma(K)$ and $u \in R\left(E_{\mu}(K)\right), \varepsilon_{h, u} \rightarrow 0$ as $h \rightarrow 0$.

Now we are ready to demonstrate the error estimate for eigenvalues. 
Theorem 2.3. Under Assumptions (A1)-(A6), suppose $\lambda$ is an eigenvalue of (2.1) with multiplicity $m$, and $\left\{u_{j}\right\}_{j=1}^{m}$ are the corresponding eigenfunctions. Then when $h$ is sufficiently small there exists $m$ eigenvalues of (2.2) $\left\{\lambda_{h, j}\right\}_{j=1}^{m}$ such that for $j=1, \cdots, m$,

$$
\left|\lambda-\lambda_{h, j}\right| \leq C\left(\varepsilon_{h, u_{j}}+e_{h, \lambda^{-1}}^{2}+e_{h, \lambda^{-1}}^{\prime 2}+\delta_{h}^{2} \gamma(h)^{-2}+\delta_{h}^{\prime 2} \gamma(h)^{-2}\right) .
$$

Proof. Suppose $\left\{u_{h, j}\right\}_{j=1}^{m}$ are the eigenfunctions of (2.2) corresponding to $\left\{\lambda_{h, j}\right\}_{j=1}^{m}$. It follows from Theorem 2.1-2.2 that there exists $\left\{u_{j}\right\}_{j=1}^{m}$ a basis of the eigenspace $R\left(E_{\lambda^{-1}}(K)\right)$ satisfying for $j=1, \cdots, m$,

$$
\begin{aligned}
\left\|u_{j}-u_{h, j}\right\|_{V} & \leq C\left(e_{h, \lambda^{-1}}+\delta_{h} \gamma(h)^{-1}\right), \\
\left\|u_{j}-u_{h, j}\right\|_{X} & \leq C\left(e_{h, \lambda^{-1}}^{\prime}+\delta_{h}^{\prime} \gamma(h)^{-1}\right) .
\end{aligned}
$$

From Lemma 2.9 we have

$$
\begin{aligned}
\left|\lambda-\lambda_{h, j}\right| \leq & \left|a\left(u_{j}, u_{j}\right)-a_{w}\left(Q_{h} u_{j}, Q_{h} u_{j}\right)\right|+a_{w}\left(u_{h, j}-Q_{h} u_{j}, u_{h, j}-Q_{h} u_{j}\right) \\
& +\lambda_{h} b\left(u_{j}-u_{h, j}, u_{j}-u_{h, j}\right) \\
\leq & C\left(\left|a\left(u_{j}, u_{j}\right)-a_{w}\left(Q_{h} u_{j}, Q_{h} u_{j}\right)\right|+\left\|u_{h, j}-Q_{h} u_{j}\right\|_{V}^{2}+\left\|u_{j}-u_{h, j}\right\|_{X}^{2}\right) \\
\leq & C\left(\left|a\left(u_{j}, u_{j}\right)-a_{w}\left(Q_{h} u_{j}, Q_{h} u_{j}\right)\right|+\left\|u_{j}-u_{h, j}\right\|_{V}^{2}+\left\|u_{j}-Q_{h} u_{j}\right\|_{V}^{2}+\left\|u_{j}-u_{h, j}\right\|_{X}^{2}\right),
\end{aligned}
$$

which implies that

$$
\left|\lambda-\lambda_{h, j}\right| \leq C\left(\varepsilon_{h, u_{j}}+e_{h, \lambda^{-1}}^{2}+e_{h, \lambda^{-1}}^{\prime 2}+\delta_{h}^{2} \gamma(h)^{-2}+\delta_{h}^{\prime 2} \gamma(h)^{-2}\right) .
$$

Hence the proof is completed.

Finally, we close this section with a lower bound estimation. To this end, we need the following assumption.

Assumption (A7). Suppose there holds $\varepsilon_{h, u} \geq \lambda_{h}\left\|u-u_{h}\right\|_{X}^{2}$ for $(\lambda, u)$ an eigenpair of (2.1) and $\left(\lambda_{h}, u_{h}\right)$ an eigenpair of $(2.2)$.

Theorem 2.4. Suppose $(\lambda, u)$ is an eigenpair of $(2.1)$ and $\left(\lambda_{h}, u_{h}\right)$ is an eigenpair of $(2.2)$, under Assumptions (A1), (A3) and (A7) we have

$$
\lambda \geq \lambda_{h}
$$

Proof. It follows from Lemma 2.9 that

$$
\begin{aligned}
& \lambda-\lambda_{h}= a(u, u)-a_{w}\left(Q_{h} u, Q_{h} u\right)+a_{w}\left(u_{h}-Q_{h} u, u_{h}-Q_{h} u\right) \\
&-\lambda_{h} b\left(u-u_{h}, u-u_{h}\right) \\
& \geq \varepsilon_{h, u}-\lambda_{h}\left\|u-u_{h}\right\|_{X}^{2} \\
& \geq 0
\end{aligned}
$$

which completes the proof. 


\section{Applications to Laplacian and biharmonic eigenvalue problems}

In this section, we solve the Laplacian eigenvalue problems and the biharmonic eigenvalue problems by the weak Galerkin method. We apply the framework in the previous section to the Laplacian eigenvalue problems and the biharmonic eigenvalue problems.

\subsection{The Laplacian eigenvalue problem}

Consider the following model problem: Find $(\lambda, u)$ such that

$$
\begin{cases}-\Delta u=\lambda u, & \text { in } \Omega, \\ u=0, & \text { on } \partial \Omega, \\ \int_{\Omega} u^{2} d \Omega=1, & \end{cases}
$$

where $\Omega$ is a polygonal or polyhedral domain in $\mathbb{R}^{d}(d=2,3)$. The variational form of problem (3.1) is defined as follows:

Find $u \in H_{0}^{1}(\Omega)$ and $\lambda \in \mathbb{R}$ such that $b(u, u)=1$ and

$$
a(u, v)=\lambda b(u, v), \quad \forall v \in H_{0}^{1}(\Omega),
$$

where

$$
a(u, v)=(\nabla u, \nabla v) \quad \text { and } \quad b(u, v)=(u, v) .
$$

It is well known that problem (3.2) has the eigenvalue sequence [3]

$$
0<\lambda_{1} \leq \lambda_{2} \leq \cdots \leq \lambda_{j} \leq \cdots \longrightarrow+\infty
$$

with the corresponding eigenfunction sequence

$$
u_{1}, u_{2}, \cdots, u_{j}, \cdots
$$

such that $b\left(u_{i}, u_{j}\right)=\delta_{i j}, i, j=1,2, \cdots$.

We denote $(\cdot, \cdot)_{m, \omega}$ and $\|\cdot\|_{m, \omega}$ the inner-product and the norm on $H^{m}(\omega)$, respectively. If the region $\omega$ is an edge or face of some elements, we use $\langle\cdot, \cdot\rangle_{m, \omega}$ instead of $(\cdot, \cdot)_{m, \omega}$. We shall drop the subscript when $m=0$ or $\omega=\Omega$. $C$ denotes a generic positive constant which is independent of the mesh size. We use $a \lesssim b$ to represent $a \leq C b$.

In what follows, we use the WG method to solve the problem (3.1). First, we introduce the WG scheme for (3.1). Then, we verify Assumptions (A1)-(A6) in Section 2 to estimate the error of the WG method. Finally, we verify (A7) to get a lower bound estimation. 


\subsubsection{The weak Galerkin scheme}

We start by introducing some notation in the WG scheme. Let $\mathcal{T}_{h}$ be a partition of the domain $\Omega$, and the elements in $\mathcal{T}_{h}$ are polygons satisfying the regular assumptions specified in [38]. Denote by $\mathcal{E}_{h}$ the edges in $\mathcal{T}_{h}$, and by $\mathcal{E}_{h}^{0}$ the interior edges $\mathcal{E}_{h} \backslash \partial \Omega$. For each element $T \in \mathcal{T}_{h}, h_{T}$ represents the diameter of $T$, and $h=\max _{T \in \mathcal{T}_{h}} h_{T}$ denotes the mesh size.

Now we introduce the WG scheme to solve the problem (3.1). For a given integer $k \geq 1$, define the WG finite element space

$$
V_{h}=\left\{v=\left\{v_{0}, v_{b}\right\}:\left.v_{0}\right|_{T} \in P_{k}(T),\left.v_{b}\right|_{e} \in P_{k-1}(e), \forall T \in \mathcal{T}_{h}, e \in \mathcal{E}_{h} \text {, and } v_{b}=0 \text { on } \partial \Omega\right\},
$$

where $P_{k}(T)$ denotes the space of polynomials on $T$ with degree no more than $k$, and $P_{k-1}(e)$ denotes the space of polynomials on $e$ with degree no more than $k-1$.

For the aim of analysis, some projection operators are also employed in this paper. Let $Q_{0}$ denote the $L^{2}$ projection from $L^{2}(T)$ onto $P_{k}(T)$ for $\forall T \in \mathcal{T}_{h}, Q_{b}$ denote the $L^{2}$ projection from $L^{2}(e)$ onto $P_{k-1}(e)$ for $\forall e \in \mathcal{E}_{h}$, and $Q_{h}$ denote the $L^{2}$ projection from $\left[L^{2}(T)\right]^{d}$ onto $\left[P_{k-1}(T)\right]^{d}$ for $\forall T \in \mathcal{T}_{h}$. Combining $Q_{0}$ and $Q_{b}$ together, we define $Q_{h}=\left\{Q_{0}, Q_{b}\right\}$, which is a projection onto $V_{h}$.

For each $v \in V_{h}$, we define its weak gradient $\nabla_{w} v$ by distribution element-wisely as follows.

Definition 3.1. For each $v \in V_{h},\left.\nabla_{w} v\right|_{T}$ is the unique polynomial in $\left[P_{k-1}(T)\right]^{d}$ satisfying

$$
\left(\nabla_{w} v, \mathbf{q}\right)_{T}=-\left(v_{0}, \nabla \cdot \mathbf{q}\right)_{T}+\left\langle v_{b}, \mathbf{q} \cdot \mathbf{n}\right\rangle_{\partial T}, \quad \forall \mathbf{q} \in\left[P_{k-1}(T)\right]^{d},
$$

where $\mathbf{n}$ denotes the outward unit normal vector.

The following commutative property for the weak gradient operator plays an essential role in the analysis. The proof can be found in [30, Lemma 5.1].

Lemma 3.1. For any $\varphi \in H^{1}(\Omega)$, there holds that on each element $T \in \mathcal{T}_{h}$

$$
\nabla_{w}\left(Q_{h} \varphi\right)=\mathbb{Q}_{h}(\nabla \varphi) .
$$

Next we define three bilinear forms on $V_{h}$. For any $v, w \in V_{h}$,

$$
\begin{aligned}
& s(v, w)=\gamma(h) \sum_{T \in \mathcal{T}_{h}} h_{T}^{-1}\left\langle Q_{b} v_{0}-v_{b}, Q_{b} w_{0}-w_{b}\right\rangle_{\partial T}, \\
& a_{w}(v, w)=\left(\nabla_{w} v, \nabla_{w} w\right)+s(v, w), \\
& b_{w}(v, w)=\left(v_{0}, w_{0}\right) .
\end{aligned}
$$

$\gamma(h)$ is a parameter selected as follows.

$$
\gamma(h)=h^{\varepsilon} \text { for small positive constant } \varepsilon,
$$

or

$$
\gamma(h)=-\frac{1}{\log (h)} .
$$

Now we are ready to state the WG algorithm. 
Weak Galerkin Algorithm 1. Find $u_{h} \in V_{h}, \lambda_{h} \in \mathbb{R}$ such that $b_{w}\left(u_{h}, u_{h}\right)=1$ and

$$
a_{w}\left(u_{h}, v\right)=\lambda_{h} b_{w}\left(u_{h}, v\right), \quad \forall v \in V_{h} .
$$

\subsubsection{Error analysis}

In this subsection, we verify Assumptions (A1)-(A6) in Section 2 to give the error estimation for the weak Galerkin method.

Denote $V_{c}=H_{0}^{1}(\Omega)$, and $V=V_{c}+V_{h}$. For any $v, w \in V$, we define the inner-product

$$
(v, w)_{V}=\left(v_{0}, w_{0}\right)+\sum_{T \in \mathcal{T}_{h}} h_{T}^{-1}\left\langle Q_{b}\left(v_{0}-v_{b}\right), Q_{b}\left(w_{0}-w_{b}\right)\right\rangle_{\partial T},
$$

where $v_{0}$ denotes the value of $v$ on the interior of each element $T \in \mathcal{T}_{h}$, and $v_{b}$ denotes the value of $v$ on the edges $\mathcal{E}_{h}$. The corresponding semi-norm is

$$
\|v\|_{V}^{2}=\sum_{T \in \mathcal{T}_{h}}\left\|v_{0}\right\|_{T}^{2}+\sum_{T \in \mathcal{T}_{h}} h_{T}^{-1}\left\|Q_{b}\left(v_{0}-v_{b}\right)\right\|_{\partial T}^{2} .
$$

Obviously $\|\cdot\|_{V}$ coincides with $|\cdot|_{1}$ on $V_{c}$ and $\|\cdot\|_{V}$ defines a norm on $V_{h}$. Thus $\|\cdot\|_{V}$ defines a norm on $V$ and $V$ is a Hilbert space.

First we verify Assumption (A1). It is easy to check that $a(\cdot, \cdot)$ and $a_{w}(\cdot, \cdot)$ defined in (3.2) and (3.6) are symmetric bounded bilinear forms on $V$, and $a(\cdot, \cdot)$ is positive definite. Hence we only need to verify the coercivity of $a_{w}(\cdot, \cdot)$.

Lemma 3.2. For any $v_{h} \in V_{h}$, the following inequality holds

$$
a_{w}\left(v_{h}, v_{h}\right) \gtrsim \gamma(h)\left\|v_{h}\right\|_{V}^{2} .
$$

Proof. From the definition of the weak gradient operator, the trace inequality and the inverse inequality, we have

$$
\begin{aligned}
& \sum_{T \in \mathcal{T}_{h}}\left(\nabla v_{0}, \nabla v_{0}\right)_{T} \\
= & -\sum_{T \in \mathcal{T}_{h}}\left(v_{0}, \nabla \cdot \nabla v_{0}\right)_{T}+\sum_{T \in \mathcal{T}_{h}}\left\langle v_{0}, \nabla v_{0} \cdot \mathbf{n}\right\rangle_{\partial T} \\
= & \sum_{T \in \mathcal{T}_{h}}\left(\nabla_{w} v_{h}, \nabla v_{0}\right)_{T}+\sum_{T \in \mathcal{T}_{h}}\left\langle Q_{b}\left(v_{0}-v_{b}\right), \nabla v_{0} \cdot \mathbf{n}\right\rangle_{\partial T} \\
\leq & \left(\sum_{T \in \mathcal{T}_{h}}\left\|\nabla_{w} v_{h}\right\|_{T}^{2}\right)^{\frac{1}{2}}\left(\sum_{T \in \mathcal{T}_{h}}\left\|\nabla v_{0}\right\|_{T}^{2}\right)^{\frac{1}{2}}+\left(\sum_{T \in \mathcal{T}_{h}} h_{T}^{-1}\left\|Q_{b}\left(v_{0}-v_{b}\right)\right\|_{\partial T}^{2}\right)^{\frac{1}{2}}\left(\sum_{T \in \mathcal{T}_{h}} h_{T}\left\|\nabla v_{0} \cdot \mathbf{n}\right\|_{\partial T}^{2}\right)^{\frac{1}{2}} \\
\leq & \left(\sum_{T \in \mathcal{T}_{h}}\left\|\nabla_{w} v_{h}\right\|_{T}^{2}\right)^{\frac{1}{2}}\left(\sum_{T \in \mathcal{T}_{h}}\left\|\nabla v_{0}\right\|_{T}^{2}\right)^{\frac{1}{2}}+\left(\sum_{T \in \mathcal{T}_{h}} h_{T}^{-1}\left\|Q_{b}\left(v_{0}-v_{b}\right)\right\|_{\partial T}^{2}\right)^{\frac{1}{2}}\left(\sum_{T \in \mathcal{T}_{h}}\left\|\nabla v_{0}\right\|_{\partial T}^{2}\right)^{\frac{1}{2}} \\
\leq & C \sum_{T \in \mathcal{T}_{h}}\left\|\nabla_{w} v_{h}\right\|_{T}^{2}+\frac{1}{4} \sum_{T \in \mathcal{T}_{h}}\left\|\nabla v_{0}\right\|_{T}^{2}+C \sum_{T \in \mathcal{T}_{h}} h_{T}^{-1}\left\|Q_{b}\left(v_{0}-v_{b}\right)\right\|_{\partial T}^{2}+\frac{1}{4} \sum_{T \in \mathcal{T}_{h}}\left\|\nabla v_{0}\right\|_{\partial T}^{2} \\
\leq & \frac{1}{2} \sum_{T \in \mathcal{T}_{h}}\left\|\nabla v_{0}\right\|_{T}^{2}+C \gamma(h)^{-1} a_{w}\left(v_{h}, v_{h}\right),
\end{aligned}
$$


which implies that

$$
\sum_{T \in \mathcal{T}_{h}}\left\|\nabla v_{0}\right\|_{T}^{2} \leq C \gamma(h)^{-1} a_{w}\left(v_{h}, v_{h}\right)
$$

Similarly, we have

$$
\sum_{T \in \mathcal{T}_{h}} h_{T}^{-1}\left\|Q_{b}\left(v_{0}-v_{b}\right)\right\|_{\partial T}^{2}=\gamma(h)^{-1} \gamma(h) \sum_{T \in \mathcal{T}_{h}} h_{T}^{-1}\left\|Q_{b}\left(v_{0}-v_{b}\right)\right\|_{\partial T}^{2} \leq C \gamma(h)^{-1} a_{w}\left(v_{h}, v_{h}\right),
$$

which completes the proof.

Next we turn to Assumption (A2). Recall that in Section 2 the operators $K$ and $K_{h}$ are defined by

$$
\begin{aligned}
& a(K f, v)=b(f, v), \quad \forall f \in V_{c}, \\
& a_{w}\left(K_{h} f_{h}, v_{h}\right)=b\left(f_{h}, v_{h}\right), \quad \forall f_{h} \in V_{h} .
\end{aligned}
$$

From the classical PDE analysis [12], when the domain $\Omega$ is convex, $\partial \Omega$ is Lipschiz, and $f \in L^{2}(\Omega)$, there exists a constant $C$ such that $\|K f\|_{H^{2}(\Omega)} \leq C\|f\|_{L^{2}(\Omega)}$. Since $H^{2}(\Omega)$ is compact embedded into $H^{1}(\Omega)$, the operator $K$ is compact. As to $K_{h}$, notice that $K_{h}$ is a bounded linear and finite ranked operator. Thus $K_{h}$ is also compact. Hence, Assumption (A2) is verified.

In the previous subsection, we define the operator $Q_{h}=\left\{Q_{0}, Q_{b}\right\}$, where $Q_{0}$ is the $L^{2}$ projection operator onto $P_{k}(T)$ on each element $T \in \mathcal{T}_{h}$ and $Q_{b}$ the $L^{2}$ projection operator onto $P_{k-1}(e)$ on each edge $e \in \mathcal{E}_{h}$. We claim that this operator $Q_{h}$ satisfies Assumption (A3). It is obvious that $Q_{h} v_{h}=v_{h}$ for any $v_{h} \in V_{h}$. Since $Q_{0}$ is the $L^{2}$ projection operator onto $P_{k}(T)$, for any $w \in V$ we have

$$
b\left(w, v_{h}\right)=\left(w_{0}, v_{0}\right)=\left(Q_{0} w_{0}, v_{0}\right)=b\left(Q_{h} w, v_{h}\right) .
$$

Thus, Assumption (A3) is verified.

In order to obtain the estimation on the eigenfunction, we still need to verify Assumptions (A4) and (A5). Let $X$ the usual $L^{2}$ space. Denote

$$
\begin{aligned}
& \delta_{h, \mu}=\sup _{\substack{u \in R\left(E_{\mu}(K)\right),\|u\|_{V}=1}}\left\|u-Q_{h} u\right\|_{V}, \\
& \delta_{h, \mu}^{\prime}=\sup _{\substack{u \in R\left(E_{\mu}(K)\right),\|u\|_{V}=1}}\left\|u-Q_{h} u\right\|_{X} .
\end{aligned}
$$

From the properties of the projection operator, we have the following estimate.

Lemma 3.3. Suppose $R\left(E_{\mu}(K)\right) \subset H^{k}(\Omega)$, then the following estimations hold

$$
\begin{aligned}
\delta_{h, \mu} & \lesssim h^{k}, \\
\delta_{h, \mu}^{\prime} & \lesssim h^{k+1} .
\end{aligned}
$$


Proof. Suppose $v \in R\left(E_{\mu}(K)\right) \subset H^{k}(\Omega)$. It follows from the trace inequality that

$$
\begin{aligned}
\left\|v-Q_{h} v\right\|_{V}^{2} & =\sum_{T \in \mathcal{T}_{h}}\left\|\nabla\left(v-Q_{0} v\right)\right\|_{T}^{2}+\sum_{T \in \mathcal{T}_{h}} h_{T}^{-1}\left\|Q_{b}\left(Q_{0} v-Q_{b} v\right)\right\|_{\partial T}^{2} \\
& =\sum_{T \in \mathcal{T}_{h}}\left\|\nabla\left(v-Q_{0} v\right)\right\|_{T}^{2}+\sum_{T \in \mathcal{T}_{h}} h_{T}^{-1}\left\|Q_{0} v-v\right\|_{\partial T}^{2} \\
& \leq \sum_{T \in \mathcal{T}_{h}}\left\|\nabla\left(v-Q_{0} v\right)\right\|_{T}^{2}+C\left(\sum_{T \in \mathcal{T}_{h}} h_{T}^{-2}\left\|Q_{0} v-v\right\|_{T}^{2}+\sum_{T \in \mathcal{T}_{h}}\left\|\nabla\left(Q_{0} v-v\right)\right\|_{T}^{2}\right) \\
& \lesssim h^{k} .
\end{aligned}
$$

From the property of the projection operator we have

$$
\left\|v-Q_{h} v\right\|_{X}=\left\|v-Q_{0} v\right\|_{L^{2}} \lesssim h^{k+1} .
$$

Then we complete the proof.

Denote

$$
\begin{aligned}
& e_{h, \mu}=\left\|\left.\left(K-K_{h} Q_{h}\right)\right|_{R\left(E_{\mu}(K)\right)}\right\|_{V}, \\
& e_{h, \mu}^{\prime}=\left\|\left.\left(\Pi_{0} K-\Pi_{0} K_{h} Q_{h}\right)\right|_{R\left(E_{\mu}(K)\right)}\right\|_{X} .
\end{aligned}
$$

Since $e_{h, \mu}$ and $e_{h, \mu}^{\prime}$ are errors of the WG method for the Poisson equation, we consider the following equation

$$
\begin{cases}-\Delta u=f, & \text { in } \Omega, \\ u=0, & \text { on } \partial \Omega,\end{cases}
$$

with $f$ is a given $L^{2}$ function. The corresponding WG scheme is to find $u_{h} \in V_{h}$ such that

$$
a_{w}\left(u_{h}, v_{h}\right)=\left(f, v_{0}\right), \quad \forall v_{h} \in V_{h} .
$$

The error estimate of (3.8) is analyzed in the Appendix (see Theorem A.1 and Theorem A.2). Then the estimations for $e_{h, \mu}$ and $e_{h, \mu}^{\prime}$ are as follows.

Lemma 3.4. Suppose $R\left(E_{\mu}(K)\right) \subset H^{k+1}(\Omega)$, then the following estimations hold

$$
\begin{aligned}
& e_{h, \mu} \lesssim \gamma(h)^{-1} h^{k}, \\
& e_{h, \mu}^{\prime} \lesssim \gamma(h)^{-1} h^{k+1} .
\end{aligned}
$$

Thus, according to Theorem 2.1 and Theorem 2.2, we have the estimations on the eigenfunctions. 
Theorem 3.1. Suppose $\lambda$ is an eigenvalue of (3.2) with multiplicity $m$, and $R\left(E_{\mu}(K)\right) \subset$ $H^{k+1}(\Omega)$ is the corresponding m-dimensional eigenspace. Suppose $\left\{\lambda_{h, j}\right\}_{j=1}^{m}$ are the eigenvalues of (3.6) approximating $\lambda$, and $\left\{u_{h, j}\right\}_{j=1}^{m}$ is a basis of the corresponding eigenspace $R\left(E_{\mu, h}\left(K_{h}\right)\right)$. Then, for any $j=1, \cdots, m$ there exists an eigenfunction $u_{j} \in R\left(E_{\mu}(K)\right)$ such that

$$
\begin{aligned}
\left\|u_{j}-u_{j, h}\right\|_{V} \lesssim \gamma(h)^{-1} h^{k}, \\
\left\|u_{j}-u_{j, h}\right\|_{X} \lesssim \gamma(h)^{-1} h^{k+1} .
\end{aligned}
$$

From (3.4)-(3.5), the parameter $\gamma(h)$ is selected to be

$$
\gamma(h)=h^{\varepsilon} \text { for small positive constant } \varepsilon,
$$

or

$$
\gamma(h)=-\frac{1}{\log (h)} .
$$

Thus the conclusions in Theorem 3.1 are

$$
\begin{aligned}
& \left\|u_{j}-u_{j, h}\right\|_{V} \lesssim h^{k-\varepsilon}, \\
& \left\|u_{j}-u_{j, h}\right\|_{X} \lesssim h^{k+1-\varepsilon},
\end{aligned}
$$

or

$$
\begin{aligned}
\left\|u_{j}-u_{j, h}\right\|_{V} \lesssim-\log (h) h^{k}, \\
\left\|u_{j}-u_{j, h}\right\|_{X} \lesssim-\log (h) h^{k+1},
\end{aligned}
$$

which are quasi-optimal estimations.

To estimate the errors of the eigenvalues, it remains to verify Assumption (A6). Denote

$$
\varepsilon_{h, u}=a(u, u)-a_{w}\left(Q_{h} u, Q_{h} u\right) .
$$

Lemma 3.5. For any $u \in H^{k+1}(\Omega)$, the following estimate holds

$$
\left|\varepsilon_{h, u}\right| \lesssim h^{2 k}
$$

Proof. It follows from Lemma 3.1 and the trace inequality that

$$
\begin{aligned}
\left|\varepsilon_{h, u}\right| & =\left|a(u, u)-a_{w}\left(Q_{h} u, Q_{h} u\right)\right| \\
& =\left|\|\nabla u\|^{2}-\sum_{T \in \mathcal{T}_{h}}\left\|\nabla_{w} Q_{h} u\right\|_{T}^{2}-s\left(Q_{h} u, Q_{h} u\right)\right| \\
& =\left|\|\nabla u\|^{2}-\sum_{T \in \mathcal{T}_{h}}\left\|Q_{h} \nabla u\right\|_{T}^{2}-\sum_{T \in \mathcal{T}_{h}} \gamma(h) h_{T}^{-1}\left\|Q_{b}\left(Q_{0} u-Q_{b} u\right)\right\|_{\partial T}^{2}\right|
\end{aligned}
$$




$$
\begin{aligned}
& \leq\left\|\nabla u-Q_{h} \nabla u\right\|_{T}^{2}+\sum_{T \in \mathcal{T}_{h}} \gamma(h) h_{T}^{-1} \| Q_{0} u-\left.u\right|_{\partial T} ^{2} \\
& \leq\left\|\nabla u-Q_{h} \nabla u\right\|_{T}^{2}+C \sum_{T \in \mathcal{T}_{h}} \gamma(h)\left(\left.h_{T}^{-2}\left\|Q_{0} u-\left.u\right|_{\partial T} ^{2}+\right\| \nabla\left(Q_{0} u-u\right)\right|_{\partial T} ^{2}\right) \\
& \lesssim h^{2 k},
\end{aligned}
$$

which completes the proof.

Eq. (3.11) verifies Assumption (A6). From Theorem 2.3 we derive the estimation on the eigenvalues.

Theorem 3.2. Suppose $\lambda$ is an eigenvalue of (3.2) with multiplicity $m$, and $R\left(E_{\mu}(K)\right) \subset$ $H^{k+1}(\Omega)$ is corresponding m-dimensional eigenspace. Suppose $\left\{\lambda_{h, j}\right\}_{j=1}^{m}$ are the eigenvalues of (3.6) approximating $\lambda$, and $\left\{u_{h, j}\right\}_{j=1}^{m}$ is a basis of the corresponding eigenspace $R\left(E_{\mu, h}\left(K_{h}\right)\right)$. Then when $h$ is sufficiently small, for any $j=1, \cdots, m$ there holds

$$
\left|\lambda-\lambda_{h, j}\right| \lesssim \gamma(h)^{-2} h^{2 k}
$$

Proof. From Theorem 2.3 we have

$$
\left|\lambda-\lambda_{h, j}\right| \leq C\left(\varepsilon_{h, u_{j}}+e_{h, \lambda^{-1}}^{2}+e_{h, \lambda^{-1}}^{\prime 2}+\delta_{h, \lambda^{-1}}^{2} \gamma(h)^{-2}+\delta_{h, \lambda^{-1}}^{\prime 2} \gamma(h)^{-2}\right) .
$$

It follows from Lemma 3.3, Lemma 3.4, and Lemma 3.5 that

$$
\begin{aligned}
& \varepsilon_{h, u_{j}} \lesssim h^{2 k}, \quad e_{h, \lambda^{-1}} \lesssim \gamma(h)^{-1} h^{k}, \\
& e_{h, \lambda^{-1}}^{\prime} \lesssim \gamma(h)^{-1} h^{k+1}, \quad \delta_{h, \lambda^{-1}} \lesssim h^{k}, \\
& \delta_{h, \lambda^{-1}}^{\prime} \lesssim h^{k+1},
\end{aligned}
$$

which implies that

$$
\left|\lambda-\lambda_{h, j}\right| \lesssim \gamma(h)^{-2} h^{2 k}
$$

The proof is completed.

From (3.4)-(3.5), the parameter $\gamma(h)$ is selected to be

$$
\gamma(h)=h^{\varepsilon} \text { for small positive constant } \varepsilon,
$$

or

$$
\gamma(h)=-\frac{1}{\log (h)} .
$$

Thus the conclusion in Theorem 3.2 is

$$
\left|\lambda-\lambda_{h, j}\right| \lesssim h^{2 k-2 \varepsilon},
$$

or

$$
\left|\lambda-\lambda_{h, j}\right| \lesssim \log (h)^{2} \gamma(h)^{-2} h^{2 k}
$$

which is a quasi-optimal estimation. 


\subsubsection{Lower bounds}

In this subsection, we prove the WG scheme (3.6) provides asymptotic lower bounds of the eigenvalues.

We prove the lower bound by verifying Assumption (A7). The following lower bound estimate is crucial in the analysis, which is proved in [24, Theorem 2.1].

Lemma 3.6. The following lower bound for the convergence rate holds for the exact eigenfunction $u$ of the eigenvalue problem (3.2)

$$
\sum_{T \in \mathcal{T}_{h}}\left\|\nabla u-\mathbb{Q}_{h} \nabla u\right\|_{T}^{2} \gtrsim C h^{2 k} .
$$

Next lemma verifies Assumption (A7).

Lemma 3.7. Suppose $(\lambda, u)$ is an eigenpair of (3.2) and $\left(\lambda_{h}, u_{h}\right)$ is an eigenpair of (3.6) approximating $(\lambda, u)$. Suppose $\gamma(h) \ll 1$, then for sufficiently small $h$ there holds

$$
\varepsilon_{h, u} \geq \lambda_{h}\left\|u-u_{h}\right\|_{X}^{2} .
$$

Proof. From Lemma 3.1, we have

$$
\begin{aligned}
\varepsilon_{h, u} & =a(u, u)-a_{w}\left(Q_{h} u, Q_{h} u\right) \\
& =\|\nabla u\|^{2}-\sum_{T \in \mathcal{T}_{h}}\left\|\nabla_{w} Q_{h} u\right\|_{T}^{2}-s\left(Q_{h} u, Q_{h} u\right) \\
& =\|\nabla u\|^{2}-\sum_{T \in \mathcal{T}_{h}}\left\|Q_{h} \nabla u\right\|_{T}^{2}-\sum_{T \in \mathcal{T}_{h}} \gamma(h) h_{T}^{-1}\left\|Q_{b}\left(Q_{0} u-Q_{b} u\right)\right\|_{\partial T}^{2} \\
& =\sum_{T \in \mathcal{T}_{h}}\left\|\nabla u-Q_{h} \nabla u\right\|_{T}^{2}-\sum_{T \in \mathcal{T}_{h}} \gamma(h) h_{T}^{-1}\left\|Q_{b}\left(Q_{0} u-Q_{b} u\right)\right\|_{\partial T}^{2} .
\end{aligned}
$$

It follows from Lemma 3.6 and the trace inequality that

$$
\begin{aligned}
& \sum_{T \in \mathcal{T}_{h}}\left\|\nabla u-Q_{h} \nabla u\right\|_{T}^{2} \gtrsim h^{2 k}, \\
& \sum_{T \in \mathcal{T}_{h}} \gamma(h) h_{T}^{-1}\left\|Q_{b}\left(Q_{0} u-Q_{b} u\right)\right\|_{\partial T}^{2} \lesssim \gamma(h) h^{2 k} .
\end{aligned}
$$

Since $\gamma(h) \ll 1$, then when $h$ is sufficiently small we have

$$
\varepsilon_{h, u} \gtrsim h^{2 k} \text {. }
$$

From Theorem 3.1 we obtain

$$
\lambda_{h}\left\|u-u_{h}\right\|_{X}^{2} \lesssim h^{2 k+2} .
$$

Thus, when $h$ is sufficiently small we have

$$
\varepsilon_{h, u} \geq \lambda_{h}\left\|u-u_{h}\right\|_{X}^{2}
$$

which completes the proof. 
Applying Theorem 2.4 we have the following lower bounds estimation.

Theorem 3.3. Suppose $(\lambda, u)$ is an eigenpair of (3.2) and $\left(\lambda_{h}, u_{h}\right)$ is an eigenpair of (3.6) approximating $(\lambda, u)$. Suppose $\gamma(h) \rightarrow 0$ as $h \rightarrow 0$, then for sufficiently small $h$ there holds

$$
\lambda \geq \lambda_{h}
$$

Remark 3.1. In the proof of Lemma 3.7, we have

$$
\begin{aligned}
& \sum_{T \in \mathcal{T}_{h}}\left\|\nabla u-Q_{h} \nabla u\right\|_{T}^{2} \gtrsim h^{2 k}, \\
& \sum_{T \in \mathcal{T}_{h}} h_{T}^{-1}\left\|Q_{b}\left(Q_{0} u-Q_{b} u\right)\right\|_{\partial T}^{2} \lesssim h^{2 k} .
\end{aligned}
$$

Thus, there exists a constant $C_{0}$ independent of $h$ such that

$$
\sum_{T \in \mathcal{T}_{h}} h_{T}^{-1}\left\|Q_{b}\left(Q_{0} u-Q_{b} u\right)\right\|_{\partial T}^{2} \leq C_{0} \sum_{T \in \mathcal{T}_{h}}\left\|\nabla u-Q_{h} \nabla u\right\|_{T}^{2} .
$$

If we take $\gamma(h) \leq C_{0}^{-1}$, we also have

$$
\varepsilon_{h, u} \gtrsim h^{2 k},
$$

and the lower bound estimation can be proved in the same way. That is to say, the condition $\gamma(h) \ll 1$ can be replaced by $\gamma(h) \leq C_{0}^{-1}$. However, it seems difficult to estimate $C_{0}$ explicitly.

\subsection{Biharmonic equation}

In this section, we apply the framework in Section 2 to the biharmonic eigenvalue problem. The proof also contains three steps. First we introduce the weak Galerkin finite element scheme for the biharmonic eigenvalue problem. Then we verify Assumptions (A1)-(A6) to estimate the error of the eigenvalues and the eigenfunctions. Finally, we verify Assumption (A7) to give a lower bound estimation. Most of the proof is similar to Section 3.1, so we just give a brief skeleton of the proof.

\subsubsection{Weak Galerkin scheme}

Consider the biharmonic eigenvalue problem

$$
\begin{cases}\Delta^{2} u=\lambda u, & \text { in } \Omega, \\ u=\frac{\partial u}{\partial n}=0, & \text { on } \partial \Omega, \\ \int_{\Omega} u^{2}=1, & \end{cases}
$$

where $\Omega$ is a polygon or polyhedral domain in $\mathbb{R}^{d}(d=2,3)$.

The weak Galerkin finite element space is defined as follows.

$$
V_{h}=\left\{\left(v_{0}, v_{b}, v_{n}\right): v_{0} \in P_{k}(T), v_{b} \in P_{k-1}(e), v_{n} \in P_{k-1}(e) \text {, and } v_{b}=v_{n}=0 \text { on } \partial \Omega\right\},
$$

where $k \geq 2$ is an integer. 
Remark 3.2. The definition of the finite element space $V_{h}$ and its norms are different from them in Subsection 3.1. For the ease of constructing the uniform framework, here we still use $V_{h}$ to represent the finite element space.

On the finite element space $V_{h}$, we define the weak Laplacian operator $\Delta_{w}$ by distribution as follows [48].

Definition 3.2. For any $v \in V_{h}, \Delta_{w} v \in P_{k-2}(T)$ is the unique polynomial satisfying on each element $T \in \mathcal{T}_{h}$,

$$
\left(\Delta_{w} v, \varphi\right)_{T}=\left(v_{0}, \Delta \varphi\right)_{T}-\left\langle v_{b}, \nabla \varphi \cdot \mathbf{n}\right\rangle_{\partial T}+\left\langle v_{n}\left(\mathbf{n}_{e} \cdot \mathbf{n}\right), \varphi\right\rangle_{\partial T}, \quad \forall \varphi \in P_{k-2}(T),
$$

where $\mathbf{n}$ is the unit outward normal vector, and $\mathbf{n}_{e}$ is the unit normal vector on each edge.

Now, we define some projection operators onto $V_{h}$. Denote $Q_{0}$ the $L^{2}$ projection onto $P_{k}(T)$ on each element $T, Q_{b}$ the $L^{2}$ projection onto $P_{k-1}(e)$ on each element $e$, and $Q_{h} v=$ $\left\{Q_{0} v, Q_{b} v, Q_{b}\left(\nabla v \cdot \mathbf{n}_{e}\right)\right\}$ is a projection operator onto $V_{h}$.

We introduce the following three bilinear forms on $V_{h}$. For any $v, w \in V_{h}$, define

$$
\begin{aligned}
s(v, w)= & \gamma(h) \sum_{T \in \mathcal{T}_{h}} h_{T}^{-3}\left\langle Q_{b} v_{0}-v_{b}, Q_{b} w_{0}-w_{b}\right\rangle_{\partial T} \\
& +\gamma(h) \sum_{T \in \mathcal{T}_{h}} h_{T}^{-1}\left\langle\nabla v_{0} \cdot \mathbf{n}_{e}-v_{n}, \nabla w_{0} \mathbf{n}_{e}-w_{n}\right\rangle_{\partial T}, \\
a_{w}(v, w)= & \left(\Delta_{w} v, \Delta_{w} w\right)+s(v, w), \\
b_{w}(v, w)= & \left(v_{0}, w_{0}\right),
\end{aligned}
$$

where $0<\gamma(h)<1$ is a parameter to be chosen.

With these preparations, we can introduce the following Weak Galerkin Algorithm for biharmonic eigenvalue problem (3.14).

Weak Galerkin Algorithm 2. Find $\left(\lambda_{h}, u_{h}\right) \in \mathbb{R} \times V_{h}$, such that $b_{w}\left(u_{h}, u_{h}\right)=1$ and

$$
a_{w}\left(u_{h}, v\right)=\lambda_{h} b_{w}\left(u_{h}, v\right), \quad \forall v \in V_{h} .
$$

\subsubsection{Error analysis}

Now we verify Assumptions (A1)-(A6) to estimate the error of the biharmonic eigenvalue problem. Denote $V_{c}=H_{0}^{2}(\Omega)$ and $V=V_{0}+V_{h}$. For any $v \in V$, define

$$
\begin{aligned}
\|v\|_{V}^{2}= & \sum_{T \in \mathcal{T}_{h}}\left(\Delta v_{0}, \Delta v_{0}\right)_{T}+\sum_{T \in \mathcal{T}_{h}} h_{T}^{-3}\left\langle Q_{b} v_{0}-v_{b}, Q_{b} v_{0}-v_{b}\right\rangle_{\partial T} \\
& +\sum_{T \in \mathcal{T}_{h}} h_{T}^{-1}\left\langle\nabla v_{0} \cdot \mathbf{n}_{e}-v_{n}, \nabla v_{0} \cdot \mathbf{n}_{e}-v_{n}\right\rangle_{\partial T} .
\end{aligned}
$$

For $v \in V_{0}=H_{0}^{2}(\Omega), v_{0}$ is the interior value of $v$ on each element $T, v_{b}$ is the trace of $v$ on $\partial T$, and $v_{n}$ is $\nabla v \cdot \mathbf{n}_{e}$. Obviously $\|\cdot\|_{V}$ defines a norm on $V$. The proof of coercivity is similar to Lemma 3.2. 
Lemma 3.8. For any $v_{h} \in V_{h}$,

$$
a_{w}\left(v_{h}, v_{h}\right) \geq \gamma(h)\left\|v_{h}\right\|_{V}^{2} .
$$

Thus, Assumption (A1) is verified.

Now we turn to Assumption (A2). Recall that in Section 2 the operators $K$ and $K_{h}$ are defined by

$$
\begin{aligned}
& a(K f, v)=b(f, v), \quad \forall f \in V_{c}, \\
& a_{w}\left(K_{h} f_{h}, v_{h}\right)=b\left(f_{h}, v_{h}\right), \quad \forall f_{h} \in V_{h} .
\end{aligned}
$$

From the regularity of the biharmonic equation, there exists a constant $C$ such that $\|K f\|_{H^{2}(\Omega)} \leq C\|f\|_{L^{2}(\Omega)}$. Since $H^{2}(\Omega)$ is compact embedded into $H^{1}(\Omega)$, the operator $K$ is compact. As to $K_{h}$, notice that $K_{h}$ is a linear bounded finite ranked operator. Thus $K_{h}$ is also compact. Hence, Assumption (A2) is verified. Also, $Q_{h}$ satisfies Assumption (A3). Hence, Assumption (A3) is verified.

In order to get the estimation on the eigenfunction, we still need to verify Assumptions (A4) and (A5). Let $X$ be the usual $L^{2}$ space. Denote

$$
\begin{gathered}
\delta_{h, \mu}=\sup _{\substack{u \in R\left(E_{\mu}(K)\right),\|u\|_{V}=1}}\left\|u-Q_{h} u\right\|_{V}, \\
\delta_{h, \mu}^{\prime}=\sup _{\substack{u \in R\left(E_{\mu}(K)\right),\|u\|_{V}=1}}\left\|u-Q_{h} u\right\|_{X} .
\end{gathered}
$$

From the properties of the projection operator, we have the following estimate.

Lemma 3.9. Suppose $R\left(E_{\mu}(K)\right) \subset H^{k}(\Omega)$, then the following estimations hold

$$
\begin{aligned}
& \delta_{h, \mu} \lesssim h^{k-1}, \\
& \delta_{h, \mu}^{\prime} \lesssim h^{k+1} .
\end{aligned}
$$

Denote

$$
\begin{aligned}
& e_{h, \mu}=\left\|\left.\left(K-K_{h} Q_{h}\right)\right|_{R\left(E_{\mu}(K)\right)}\right\|_{V}, \\
& e_{h, \mu}^{\prime}=\left\|\left.\left(\Pi_{0} K-\Pi_{0} K_{h} Q_{h}\right)\right|_{R\left(E_{\mu}(K)\right)}\right\|_{X} .
\end{aligned}
$$

The estimation for $e_{h, \mu}$ and $e_{h, \mu}^{\prime}$ is similar to the proof of Theorem 4.2 and Theorem 5.1 in [48].

Lemma 3.10. Suppose $R\left(E_{\mu}(K)\right) \subset H^{k+2}(\Omega)$, then the following estimations hold

$$
\begin{aligned}
& e_{h, \mu} \lesssim \gamma(h)^{-1} h^{k-1}, \\
& e_{h, \mu}^{\prime} \lesssim \gamma(h)^{-1} h^{k+k_{0}-2},
\end{aligned}
$$

where $k_{0}=\min \{k, 3\}$. 
Now we give the estimates for the eigen-pair of problem (3.14) based on the previous results.

Theorem 3.4. Suppose $\lambda$ is an eigenvalue of (3.14) with multiplicity $m$, and $R\left(E_{\mu}(K)\right) \subset$ $H^{k+2}(\Omega)$ is corresponding m-dimensional eigenspace. Suppose $\left\{\lambda_{h, j}\right\}_{j=1}^{m}$ are the eigenvalues of (3.6) approximating $\lambda$, and $\left\{u_{h, j}\right\}_{j=1}^{m}$ is a basis of the corresponding eigenspace $R\left(E_{\mu, h}\left(K_{h}\right)\right)$. Then, for any $j=1, \cdots, m$ there exists an eigenfunction $u_{j} \in R\left(E_{\mu}(K)\right)$ such that

$$
\begin{aligned}
& \left\|u_{j}-u_{j, h}\right\|_{V} \lesssim \gamma(h)^{-1} h^{k-1}, \\
& \left\|u_{j}-u_{j, h}\right\|_{X} \lesssim \gamma(h)^{-1} h^{k+k_{0}-2},
\end{aligned}
$$

where $k_{0}=\min \{k, 3\}$.

For the estimation of the eigenvalues, Assumption (A6) is still remained to be verified. Denote

$$
\varepsilon_{h, u}=a(u, u)-a_{w}\left(Q_{h} u, Q_{h} u\right) .
$$

Lemma 3.11. For any $u \in H^{k+2}(\Omega)$, the following estimate holds

$$
\left|\varepsilon_{h, u}\right| \lesssim h^{2 k-2}
$$

Then we derive the estimation on the eigenvalues.

Theorem 3.5. Suppose $\lambda$ is an eigenvalue of (3.2) with multiplicity $m$, and $R\left(E_{\mu}(K)\right) \subset$ $H^{k+2}(\Omega)$ is corresponding m-dimensional eigenspace. Suppose $\left\{\lambda_{h, j}\right\}_{j=1}^{m}$ are the eigenvalues of (3.6) approximating $\lambda$, and $\left\{u_{h, j}\right\}_{j=1}^{m}$ is a basis of the corresponding eigenspace $R\left(E_{\mu, h}\left(K_{h}\right)\right)$. Then when $h$ is sufficiently small, for any $j=1, \cdots, m$ there holds

$$
\left|\lambda-\lambda_{h, j}\right| \lesssim \gamma(h)^{-2} h^{2 k-2} .
$$

Similar to the discussion in Section 3.1.2, the conclusions in Theorem 3.4 and Theorem 3.5 are quasi-optimal.

\subsubsection{Lower bounds}

In this subsection, we prove the WG scheme (3.15) provides asymptotic lower bounds of the eigenvalues.

We prove the lower bound by verifying Assumption (A7). The following lower bound estimate is crucial in the analysis, which is proved in [24, Theorem 2.1].

Lemma 3.12. The following lower bound for the convergence rate holds for the exact eigenfunction $u$ of the eigenvalue problem (3.14)

$$
\sum_{T \in \mathcal{T}_{h}}\left\|\nabla u-\mathbb{Q}_{h} \nabla u\right\|_{T}^{2} \gtrsim C h^{2 k-2} .
$$


Similar to Lemma 3.7, we have the following estimation.

Lemma 3.13. Suppose $(\lambda, u)$ is an eigenpair of (3.14) and $\left(\lambda_{h}, u_{h}\right)$ is an eigenpair of (3.15) approximating $(\lambda, u)$. Suppose $\gamma(h) \ll 1$, then for sufficiently small $h$ there holds

$$
\varepsilon_{h, u} \geq \lambda_{h}\left\|u-u_{h}\right\|_{X}^{2} .
$$

Applying Theorem 2.4 we have the following lower bounds estimation.

Theorem 3.6. Suppose $(\lambda, u)$ is an eigenpair of (3.14) and $\left(\lambda_{h}, u_{h}\right)$ is an eigenpair of (3.15) approximating $(\lambda, u)$. Suppose $\gamma(h) \rightarrow 0$ as $h \rightarrow 0$, then for sufficiently small $h$ there holds

$$
\lambda \geq \lambda_{h}
$$

\section{Numerical experiments}

In this section, we shall present some numerical results for the weak Galerkin method analyzed in the previous sections.

\subsection{Laplacian eigenvalue problem on unit square domain}

In the first example, we consider the problem (3.1) on the square domain $\Omega=(0,1)^{2}$. It has the analytic solution

$$
\lambda=\left(m^{2}+n^{2}\right) \pi^{2}, \quad u=\sin (m \pi x) \sin (n \pi y),
$$

where $m, n$ are arbitrary integers. The first six eigenvalues are $\lambda_{1}=2 \pi^{2}, \lambda_{2}=\lambda_{3}=5 \pi^{2}$, $\lambda_{4}=8 \pi^{2}$ and $\lambda_{5}=\lambda_{6}=10 \pi^{2}$.

The uniform mesh is applied in this example, and $h$ denotes the mesh size. The degree of polynomial $k=1$ and the parameter $\gamma(h)$ is selected to be $h^{0.1}$ and $-1 / \log (h)$, separately. The corresponding numerical results for the first six eigenvalues and the first six eigenfunctions are showed in Figs. 1-2. From these figures, we find the errors of eigenvalues are of approximately second order convergence, the errors of eigenfunctions under $V$-norm are of first order convergence and the $L^{2}$ error of eigenfunctions are of second order convergence, which reveal the convergence results in Theorem 3.2 and Theorem 3.1.

\subsection{Rectangular mesh}

In this example, we show that the weak Galerkin method is valid on different type of meshes. We still test the algorithm by solving the Laplacian eigenvalue problem on the unit square domain. The square mesh is employed, and the degree of polynomial is $k=1$. The parameter $\gamma(h)=h^{0.1}$. The numerical results for the first six eigenvalues are listed in Table 1. Table 1 shows that all the approximate eigenvalues are the lower bounds of the exact eigenvalues, which is predicted in Theorem 3.3. The convergence rates are coincide with Theorem 3.2. 


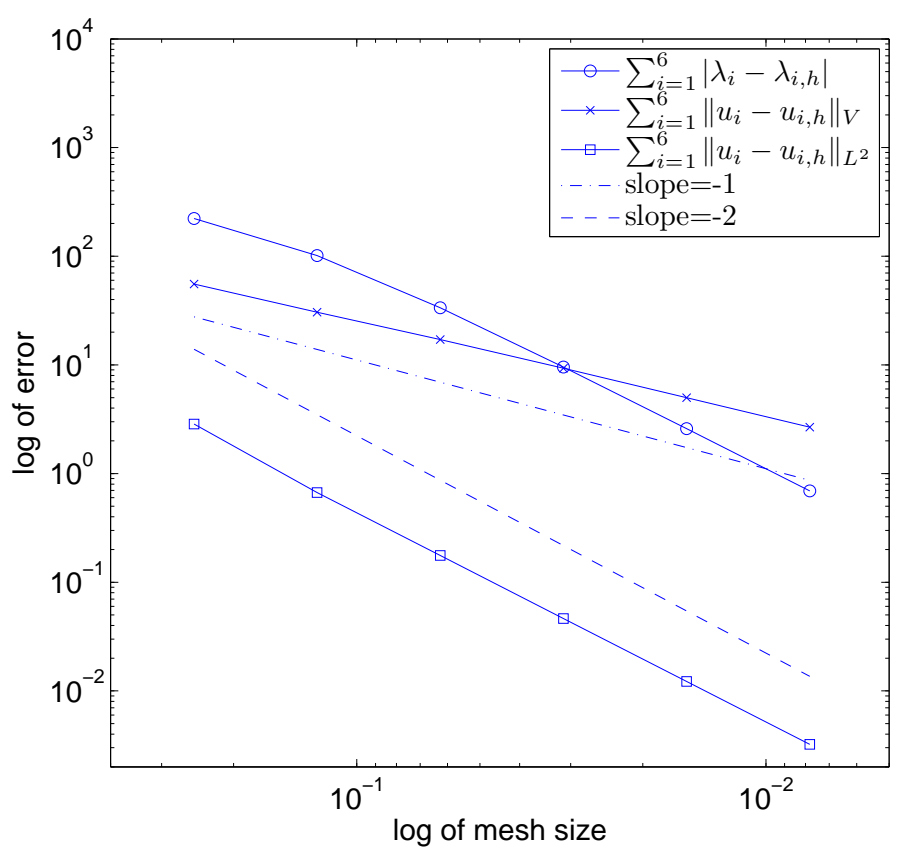

Figure 1: Errors for case $\gamma(h)=h^{0.1}$.

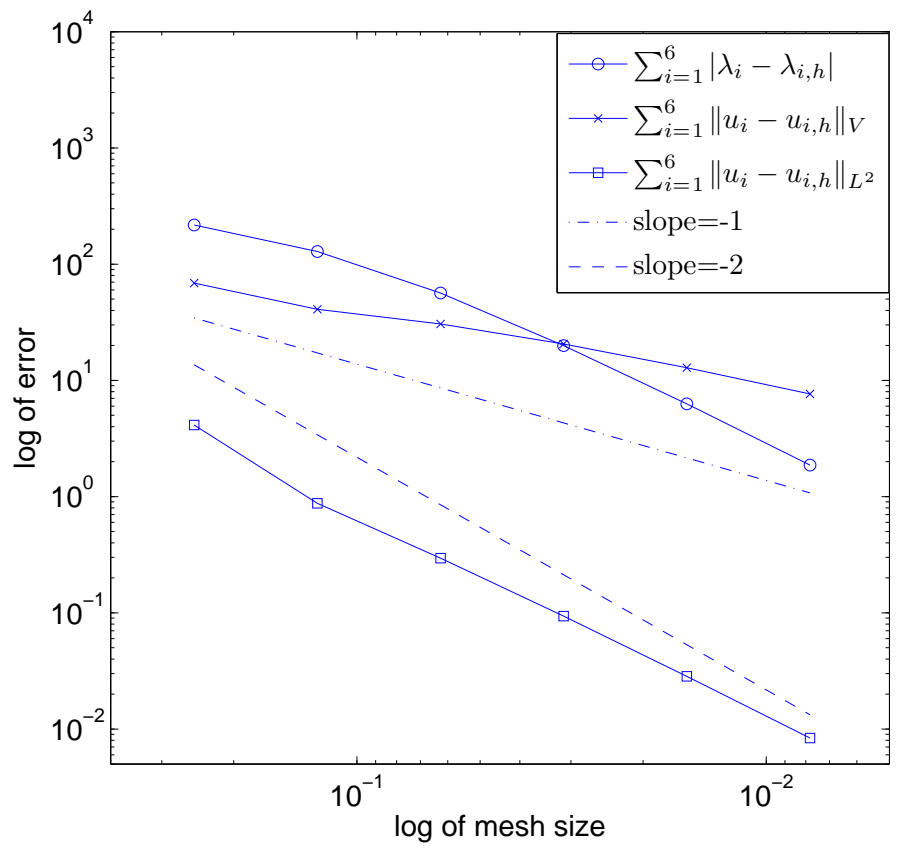

Figure 2: Errors for case $\gamma(h)=-1 / \log (h)$. 
Table 1: Errors of eigenvalues for rectangular mesh.

\begin{tabular}{||c|c|c|c|c|c||}
\hline$h$ & $1 / 8$ & $1 / 16$ & $1 / 32$ & $1 / 64$ & $1 / 128$ \\
\hline \hline$\lambda_{1}-\lambda_{1, h}$ & $1.7000 \mathrm{e}+0$ & $4.8873 \mathrm{e}-1$ & $1.3355 \mathrm{e}-1$ & $3.5976 \mathrm{e}-2$ & $9.6533 \mathrm{e}-3$ \\
\hline order & & 1.7984 & 1.8716 & 1.8923 & 1.8979 \\
\hline$\lambda_{2}-\lambda_{2, h}$ & $1.0980 \mathrm{e}+1$ & $3.6389 \mathrm{e}+0$ & $1.0456 \mathrm{e}+0$ & $2.8660 \mathrm{e}-1$ & $7.7494 \mathrm{e}-2$ \\
\hline order & & 1.5933 & 1.7992 & 1.8672 & 1.8869 \\
\hline$\lambda_{3}-\lambda_{3, h}$ & $1.0980 \mathrm{e}+1$ & $3.6389 \mathrm{e}+0$ & $1.0456 \mathrm{e}+0$ & $2.8660 \mathrm{e}-1$ & $7.7494 \mathrm{e}-2$ \\
\hline order & & 1.5933 & 1.7992 & 1.8672 & 1.8869 \\
\hline$\lambda_{4}-\lambda_{4, h}$ & $2.1311 \mathrm{e}+1$ & $7.2430 \mathrm{e}+0$ & $2.0915 \mathrm{e}+0$ & $5.7228 \mathrm{e}-1$ & $1.5421 \mathrm{e}-1$ \\
\hline order & & 1.5569 & 1.7921 & 1.8697 & 1.8918 \\
\hline$\lambda_{5}-\lambda_{5, h}$ & $3.8500 \mathrm{e}+1$ & $1.5266 \mathrm{e}+1$ & $4.7322 \mathrm{e}+0$ & $1.3302 \mathrm{e}+0$ & $3.6275 \mathrm{e}-1$ \\
\hline order & & 1.3346 & 1.6897 & 1.8309 & 1.8746 \\
\hline$\lambda_{6}-\lambda_{6, h}$ & $3.8500 \mathrm{e}+1$ & $1.5266 \mathrm{e}+1$ & $4.7322 \mathrm{e}+0$ & $1.3302 \mathrm{e}+0$ & $3.6275 \mathrm{e}-1$ \\
\hline order & & 1.3346 & 1.6897 & 1.8309 & 1.8746 \\
\hline
\end{tabular}

\subsection{High order element}

In this example, we show that the weak Galerkin method produces high order lower bounds of the eigenvalues. We still solve the Laplacian eigenvalue problem on the unit square domain. The uniform triangular mesh is employed, and the degree of polynomial is $k=4$. The parameter is $\gamma(h)=h^{0.1}$. The numerical results for the first six eigenvalues are listed in Table 2. Table 2 shows that all the approximate eigenvalues are the lower bounds of the exact eigenvalues, which is predicted in Theorem 3.3. The convergence rates are coincide with Theorem 3.2.

\subsection{Laplacian eigenvalue problem on L shape domain}

Now we consider the eigenvalue problem (3.1) on the $L$ shape domain $\Omega=(-1,1)^{2} \backslash[0,1)^{2}$.

We also use the weak Galerkin method to solve this eigenvalue problem and Table 3 presents the corresponding numerical results for the first six eigenvalues. In this example, the degree of polynomial is $k=2$, and the parameter $\gamma(h)=h^{0.1}$. The uniform triangular mesh is employed. Even the analytic eigenpairs are not known, from the table we find the numerical eigenvalues $\lambda_{j, h}$ increase when $h$ decreases which shows that $\lambda_{j, h}$ are lower bounds of the exact eigenvalue $\lambda_{j}$.

\subsection{Biharmonic eigenvalue problem on unit square domain}

We consider the problem (3.14) on the square domain $\Omega=(0,1)^{2}$. The first eigenvalue is 1294.9339598. 
Table 2: Errors of eigenvalues for $k=4$.

\begin{tabular}{||c|c|c|c|c||}
\hline$h$ & $1 / 2$ & $1 / 4$ & $1 / 8$ & $1 / 16$ \\
\hline \hline$\lambda_{1}-\lambda_{1, h}$ & $9.4729 \mathrm{e}-3$ & $3.2299 \mathrm{e}-5$ & $1.3058 \mathrm{e}-7$ & $5.3536 \mathrm{e}-10$ \\
\hline order & & 8.1962 & 7.9504 & 7.9303 \\
\hline$\lambda_{2}-\lambda_{2, h}$ & $5.6202 \mathrm{e}+0$ & $3.9834 \mathrm{e}-3$ & $1.4516 \mathrm{e}-5$ & $5.9074 \mathrm{e}-8$ \\
\hline order & & 10.4624 & 8.1002 & 7.9409 \\
\hline$\lambda_{3}-\lambda_{3, h}$ & $3.7210 \mathrm{e}+0$ & $1.9021 \mathrm{e}-3$ & $7.2664 \mathrm{e}-6$ & $2.9831 \mathrm{e}-8$ \\
\hline order & & 10.9339 & 8.0322 & 7.9283 \\
\hline$\lambda_{4}-\lambda_{4, h}$ & $3.3030 \mathrm{e}+1$ & $4.2515 \mathrm{e}-2$ & $1.3976 \mathrm{e}-4$ & $5.6171 \mathrm{e}-7$ \\
\hline order & & 9.6016 & 8.2489 & 7.9589 \\
\hline$\lambda_{5}-\lambda_{5, h}$ & $5.2616 \mathrm{e}+1$ & $1.1007 \mathrm{e}-1$ & $3.0953 \mathrm{e}-4$ & $1.2189 \mathrm{e}-6$ \\
\hline order & & 8.9010 & 8.4741 & 7.9884 \\
\hline$\lambda_{6}-\lambda_{6, h}$ & $5.2223 \mathrm{e}+1$ & $1.0988 \mathrm{e}-1$ & $3.0953 \mathrm{e}-4$ & $1.2189 \mathrm{e}-6$ \\
\hline order & & 8.8927 & 8.4716 & 7.9884 \\
\hline
\end{tabular}

Table 3: Discrete eigenvalues for $\gamma(h)=h^{0.1}$ and $k=2$.

\begin{tabular}{||c|c|c|c|c|c|c||}
\hline$h$ & $1 / 4$ & $1 / 8$ & $1 / 16$ & $1 / 32$ & $1 / 64$ & $1 / 128$ \\
\hline \hline$\lambda_{1, h}$ & 9.5538 & 9.6152 & 9.6306 & 9.6362 & 9.6383 & 9.6392 \\
\hline$\lambda_{2, h}$ & 15.0957 & 15.1903 & 15.1967 & 15.1972 & 15.1972 & 15.1973 \\
\hline$\lambda_{3, h}$ & 19.5148 & 19.7251 & 19.7383 & 19.7391 & 19.7392 & 19.7392 \\
\hline$\lambda_{4, h}$ & 28.7653 & 29.4755 & 29.5185 & 29.5213 & 29.5215 & 29.5215 \\
\hline$\lambda_{5, h}$ & 30.6553 & 31.7870 & 31.8860 & 31.9036 & 31.9091 & 31.9113 \\
\hline$\lambda_{6, h}$ & 39.0485 & 41.2933 & 41.4490 & 41.4674 & 41.4719 & 41.4735 \\
\hline
\end{tabular}

The nonuniform triangular mesh is applied in this example. The degree of polynomial $k=3$ and $\gamma(h)$ is selected to be 1 and $h^{0.5}$, separately. The corresponding numerical results for the first eigenvalue are showed in Table 4. From the table, we find the weak Galerkin method gives the reasonable numerical approximations. Furthermore, the choice of $\varepsilon$ really affects the convergence order which means the convergence results in Theorem 3.5 are also reasonable. The numerical results included in Table 4 show the eigenvalue approximation $\lambda_{j, h}$ are lower bounds of the exact eigenvalue $\lambda_{j}$, which reveal the convergence results in Theorem 3.5.

\section{Concluding remarks}

In this paper, we apply the weak Galerkin method to solve the eigenvalue problems and present the corresponding convergence analysis. Furthermore, we analyze the lowerbound property of the weak Galerkin method. Compared with the classical noncon- 
Table 4: Error for the first eigenvalue $\lambda_{1}-\lambda_{1, h}$.

\begin{tabular}{||c|c|c|c|c|c|c||}
\hline & \multicolumn{3}{|c|}{$\gamma(h)=1$} & \multicolumn{3}{c||}{$\gamma(h)=h^{0.5}$} \\
\hline$h$ & $\lambda_{1, h}$ & $\lambda_{1}-\lambda_{1, h}$ & order & $\lambda_{1, h}$ & $\lambda_{1}-\lambda_{1, h}$ & order \\
\hline $1 / 20$ & 1276.5378 & $1.8396 \mathrm{e}+1$ & & 1231.0779 & $6.3856 \mathrm{e}+1$ & \\
\hline $1 / 40$ & 1293.6901 & $1.2438 \mathrm{e}+0$ & 3.8865 & 1288.5256 & $6.4083 \mathrm{e}+0$ & 3.3168 \\
\hline $1 / 60$ & 1294.6810 & $2.5289 \mathrm{e}-1$ & 3.9288 & 1293.3300 & $1.6040 \mathrm{e}+0$ & 3.4162 \\
\hline $1 / 80$ & 1294.8528 & $8.1142 \mathrm{e}-2$ & 3.9514 & 1294.3363 & $5.9758 \mathrm{e}-1$ & 3.4320 \\
\hline $1 / 100$ & 1294.9004 & $3.3478 \mathrm{e}-2$ & 3.9675 & 1294.6568 & $2.7712 \mathrm{e}-1$ & 3.4438 \\
\hline $1 / 120$ & 1294.9177 & $1.6206 \mathrm{e}-2$ & 3.9792 & 1294.7863 & $1.4763 \mathrm{e}-1$ & 3.4540 \\
\hline
\end{tabular}

forming finite element method which provides lower bound approximation by linear element with only the second order convergence, the weak Galerkin method presents lower bound approximations with arbitrary order convergence. These results can be extended to general elliptic operators along similar lines.

Based on the WG scheme for the eigenvalue problems, some acceleration technique, such as the two-grid method [41] and the shifted-inverse power method [40], can be applied to the WG scheme to reduce the computational cost. Upper bounds of the eigenvalues can also be produce by the WG method with a interpolation technique [42]. In the future, we plan to design efficient solvers for the algebraic eigenvalue problems derived by the weak Galerkin method by using multigrid precondition. We also plan to speed up our numerical algorithm by the shifting method, etc.

\section{Acknowledgments}

The research of Ran Zhang was supported in part by China Natural National Science Foundation (91630201, U1530116, 11771179), and by the Program for Cheung Kong Scholars of Ministry of Education of China, Key Laboratory of Symbolic Computation and Knowledge Engineering of Ministry of Education, Jilin University, Changchun, 130012, P.R. China. The research of Zhimin Zhang was supported in part by the National Natural Science Foundation of China (NSFC 11471031, 91430216) and the U.S. National Science Foundation (DMS-1419040). The research of Hehu Xie was supported by Science Challenge Project (No. TZ2016002), National Natural Science Foundations of China (NSFC 11771434, 91330202, 11371026, 91430108, 11771322, 11626033, 11601368), the National Center for Mathematics and Interdisciplinary Science, CAS.

\section{Appendix}

In this section, we present some technique tools used in the previous sections. 
Consider the Poisson equation

$$
\begin{cases}-\Delta u=f, & \text { in } \Omega, \\ u=0, & \text { on } \partial \Omega,\end{cases}
$$

with $f$ is a given $L^{2}$ function. The corresponding weak Galerkin scheme is to find $u_{h} \in V_{h}$ such that

$$
a_{w}\left(u_{h}, v_{h}\right)=\left(f, v_{0}\right), \quad \forall v_{h} \in V_{h} .
$$

Suppose $u$ is the solution of (3.7), and $u_{h}$ is the numerical solution of (3.8). Denote by $e_{h}$ the error that

$$
e_{h}=Q_{h} u-u_{h}=\left\{Q_{0} u-u_{0}, Q_{b} u-u_{b}\right\} .
$$

Then $e_{h}$ satisfies the following equation.

Lemma A.1. Let $e_{h}$ be the error of the weak Galerkin scheme (3.8). Then, for any $v \in V_{h}$, we have

$$
a_{w}\left(e_{h}, v\right)=\ell(u, v)+s\left(Q_{h} u, v\right)
$$

where

$$
\ell(u, v)=\sum_{T \in \mathcal{T}_{h}}\left\langle\left(\nabla u-\mathbb{Q}_{h} \nabla u\right) \cdot \mathbf{n}, v_{0}-v_{b}\right\rangle_{\partial T} .
$$

Proof. From the definition of the weak gradient (3.3) and the commutative property (3.1), we obtain on each element $T \in \mathcal{T}_{h}$ that

$$
\begin{aligned}
\left(\nabla_{w} Q_{h} u, \nabla_{w} v\right)_{T} & =\left(\mathbb{Q}_{h} \nabla u, \nabla_{w} v\right)_{T} \\
& =-\left(v_{0}, \nabla \cdot \mathbb{Q}_{h} \nabla u\right)_{T}+\left\langle v_{b}, \mathbb{Q}_{h}(\nabla u) \cdot \mathbf{n}\right\rangle_{\partial T} \\
& =\left(\nabla v_{0}, \mathbb{Q}_{h} \nabla u\right)_{T}-\left\langle v_{0}-v_{b}, \mathbb{Q}_{h}(\nabla u) \cdot \mathbf{n}\right\rangle_{\partial T} \\
& =\left(\nabla v_{0}, \nabla u\right)_{T}-\left\langle\mathbb{Q}_{h}(\nabla u) \cdot \mathbf{n}, v_{0}-v_{b}\right\rangle_{\partial T} .
\end{aligned}
$$

Summing over all elements and it follows that

$$
\begin{aligned}
\left(\nabla_{w} Q_{h} u, \nabla_{w} v\right) & =\left(\nabla v_{0}, \nabla u\right)-\sum_{T \in \mathcal{T}_{h}}\left\langle\mathbf{Q}_{h}(\nabla u) \cdot \mathbf{n}, v_{0}-v_{b}\right\rangle_{\partial T} \\
& =\left(f, v_{0}\right)+\sum_{T \in \mathcal{T}_{h}}\left\langle\nabla u \cdot \mathbf{n}, v_{0}\right\rangle_{\partial T}-\sum_{T \in \mathcal{T}_{h}}\left\langle\mathbf{Q}_{h}(\nabla u) \cdot \mathbf{n}, v_{0}-v_{b}\right\rangle_{\partial T} \\
& =\left(f, v_{0}\right)+\ell(u, v) .
\end{aligned}
$$

Notice that the numerical solution $u_{h}$ satisfies (3.8). Then we derive that

$$
a_{w}\left(e_{h}, v\right)=\ell(u, v)+s\left(Q_{h} u, v\right), \quad \forall v \in V_{h},
$$

which completes the proof. 
With the tools above we give the estimates for $\ell(w, v)$ and $s\left(Q_{h} w, v\right)$ as follows.

Lemma A.2. For any $v_{h} \in V_{h}$ and $w \in H^{k+1}(\Omega)$, the following estimates hold true,

$$
\begin{aligned}
& \left|s\left(Q_{h} w, v_{h}\right)\right| \leq C \gamma(h) h^{k}\|w\|_{k+1}\left\|v_{h}\right\|_{V}, \\
& \left|\ell\left(w, v_{h}\right)\right| \leq C h^{k}\|w\|_{k+1}\left\|v_{h}\right\|_{V} .
\end{aligned}
$$

Proof. From the Cauchy-Schwarz inequality, we obtain

$$
\begin{aligned}
\left|s\left(Q_{h} w, v\right)\right| & =\left|\gamma(h) \sum_{T \in \mathcal{T}_{h}} h_{T}^{-1}\left\langle Q_{b} Q_{0} w-Q_{b} w, Q_{b} v_{0}-v_{b}\right\rangle_{\partial T}\right| \\
& \leq C \gamma(h)\left(\sum_{T \in \mathcal{T}_{h}} h_{T}^{-1}\left\|Q_{0} w-w\right\|_{\partial T}^{2}\right)^{\frac{1}{2}}\left(\sum_{T \in \mathcal{T}_{h}} h_{T}^{-1}\left\|Q_{b} v_{0}-v_{b}\right\|_{\partial T}^{2}\right)^{\frac{1}{2}} \\
& \leq C_{\gamma}(h) h^{k}\|w\|_{k+1}\left\|v_{h}\right\|_{V} .
\end{aligned}
$$

Similarly, for the second term we derive that

$$
\begin{aligned}
|\ell(w, v)| & =\left|\sum_{T \in \mathcal{T}_{h}}\left\langle\left(\nabla w-\mathbf{Q}_{h} \nabla w\right) \cdot \mathbf{n}, v_{0}-v_{b}\right\rangle_{\partial T}\right| \\
& \leq C\left(\sum_{T \in \mathcal{T}_{h}} h_{T}\left\|\nabla w-\mathbf{Q}_{h} \nabla w\right\|_{\partial T}^{2}\right)^{\frac{1}{2}}\left(\sum_{T \in \mathcal{T}_{h}} h_{T}^{-1}\left\|v_{0}-v_{b}\right\|_{\partial T}^{2}\right)^{\frac{1}{2}} \\
& \leq C h^{k}\|w\|_{k_{0}+1}\left\|v_{h}\right\|_{V},
\end{aligned}
$$

which completes the proof.

With the error equation (A.3) and the estimates derived in Lemma A.2, we get the following error estimate for the weak Galerkin method.

Theorem A.1. Assume the exact solution of (3.7), $u \in H^{k+1}(\Omega)$, and $u_{h}$ is the numerical solution of the weak Galerkin scheme (3.8). Then the following estimates hold true,

$$
\left\|u-u_{h}\right\|_{V} \leq C_{\gamma}(h)^{-1} h^{k}\|u\|_{k+1} .
$$

Proof. Taking $v=e_{h}$ in (A.3) and it follows from Lemma 3.2 that

$$
\begin{aligned}
\gamma(h)\left\|e_{h}\right\|_{V}^{2} & \leq C a_{w}\left(e_{h}, e_{h}\right) \\
& =\ell\left(u, e_{h}\right)+s\left(Q_{h} u, e_{h}\right) \\
& \leq C h^{k}\|u\|_{k+1}\left\|e_{h}\right\|_{V}+C \gamma(h) h^{k}\|u\|_{k+1}\left\|e_{h}\right\|_{V} \\
& \leq C h^{k}\|u\|_{k+1}\left\|e_{h}\right\|_{V}
\end{aligned}
$$


which implies that

$$
\left\|e_{h}\right\|_{V} \leq C \gamma(h)^{-1} h^{k}\|u\|_{k+1}
$$

From Lemma 3.3 we have

$$
\left\|u-u_{h}\right\|_{V} \leq\left\|u-Q_{h} u\right\|_{V}+\left\|Q_{h} u-u_{h}\right\|_{V} \leq C \gamma(h)^{-1} h^{k}\|u\|_{k+1} .
$$

Thus, the proof is completed.

By using the classical Nistche's technique, we also have the $L^{2}$ error estimate.

Theorem A.2. Assume the exact solution of (3.7) $u \in H^{k+1}(\Omega)$, the dual problem of (3.7) has $H^{2}(\Omega)$-regularity, and $u_{h}$ is the numerical solution of the weak Galerkin scheme (3.8). Then the following estimates hold true,

$$
\left\|u-u_{h}\right\|_{X} \leq C_{\gamma}(h)^{-1} h^{k+1}\|u\|_{k+1} .
$$

\section{References}

[1] W. Arendt, R. NitTKA, W. Peter, AND F. Steiner, Weyl's Law: spectral properties of the Laplacian in mathematics and physics, in Math. Anal. Evol. Information, Complex., Wiley-VCH Verlag GmbH \& Co. KGaA, Weinheim, Germany, pp. 1-71.

[2] M. G. ARMENTANO AND R. G. DuRAN, Asymptotic lower bounds for eigenvalues by nonconforming finite element methods., ETNA, Electron. Trans. Numer. Anal., 17 (2004), pp. 93-101.

[3] I. BABUvsKA AND J. Osborn, Eigenvalue problems, in Handb. Numer. Anal. Vol. II, Handb. Numer. Anal., II, North-Holland, Amsterdam, 1991, pp. 641-787.

[4] D. Boffi, Finite element approximation of eigenvalue problems, Acta Numer., 19 (2010), pp. 1 120.

[5] C. Carstensen And J. Gedicke, Guaranteed lower bounds for eigenvalues, Math. Comput., 83 (2014), pp. 2605-2629.

[6] F. CHATELIN, Spectral approximation of linear operators, in Spectr. Approx. Linear Oper., Society for Industrial and Applied Mathematics, jan 2011, pp. xxvii+458.

[7] I. CHAVEL, Eigenvalues in Riemannian Geometry, vol. 53, 1989.

[8] G. Chen, M. Feng, AND X. XIE, Robust globally divergence-free weak Galerkin methods for Stokes equations, J. Comput. Math., 34 (2016), pp. 549-572.

[9] K. A. Cliffe, E. J. C. HAll, AND P. Houston, Adaptive discontinuous Galerkin methods for eigenvalue problems arising in incompressible fluid flows, SIAM J. Sci. Comput., 31 (2010), pp. 4607-4632.

[10] B. DEKA, A weak Galerkin finite element method for elliptic interface problems with polynomial reduction, Numer. Math. Theory, Methods Appl., 11 (2018), pp. 655-672.

[11] Y. DU AND Z. ZHANG, A numerical analysis of the weak Galerkin method for the Helmholtz equation with high wave number, Commun. Comput. Phys., 22 (2017), pp. 133-156.

[12] L. Evans, Partial Differential Equations, vol. 19 of Graduate Studies in Mathematics, American Mathematical Society, Providence, Rhode Island, mar 2010.

[13] K. FENG, Difference scheme based on variational principle, Appl. Math. Comput. Math, 2 (1965), pp. 238-262. 
[14] D. S. GREBENKOV AND B.-T. NGUYEN, Geometrical structure of Laplacian eigenfunctions, SIAM Rev., 55 (2013), pp. 601-667.

[15] J. K. HAle, Eigenvalues and Perturbed Domains, in Ten Math. Essays Approx. Anal. Topol., 2005, pp. 95-123.

[16] J. HU, Y. HUANG, AND Q. LIN, Lower bounds for eigenvalues of elliptic operators: By nonconforming finite element methods, J. Sci. Comput., 61 (2014), pp. 196-221.

[17] J. HU, Y. HUANG, AND Q. SHEN, Constructing both lower and upper bounds for the eigenvalues of elliptic operators by nonconforming finite element methods, Numer. Math., 131 (2014), pp. 273302.

[18] W. HuAng AND Y. WANG, Discrete maximum principle for the weak Galerkin method for anisotropic diffusion problems, Commun. Comput. Phys., 18 (2015), pp. 65-90.

[19] F. KIKUCHI AND X. LIU, Estimation of interpolation error constants for the P0 and P1 triangular finite elements, Comput. Methods Appl. Mech. Eng., 196 (2007), pp. 3750-3758.

[20] P. KuCHment, On some spectral problems of mathematical physics, Partial Differ. Equations Inverse Probl. Proc. Pan-American Adv. Stud. Inst., 0000 (2004), pp. 241-276.

[21] J. R. Kuttler AND V. G. Sigillito, Eigenvalues of the Laplacian in two dimensions, SIAM Rev., 26 (1984), pp. 163-193.

[22] M. G. LARSON, A posteriori and a priori error analysis for finite element approximations of selfadjoint elliptic eigenvalue problems, SIAM J. Numer. Anal., 38 (2000), pp. 608-625.

[23] Q. LIN, H. HUANG, AND Z. LI, New expansions of numerical eigenvalues by nonconforming elements, Math. Comput., 77 (2008), pp. 2061-2084.

[24] Q. LIN, H. XIE, AND J. XU, Lower bounds of the discretization error for piecewise polynomials, Math. Comput., 83 (2013), pp. 1-13.

[25] X. LIU, A framework of verified eigenvalue bounds for self-adjoint differential operators, Appl. Math. Comput., 267 (2015), pp. 341-355.

[26] X. LIU AND S. I. OISHI, Verified eigenvalue evaluation for the Laplacian over polygonal domains of arbitrary shape, SIAM J. Numer. Anal., 51 (2013), pp. 1634-1654.

[27] F. LUO, Q. LIN, AND H. XIE, Computing the lower and upper bounds of Laplace eigenvalue problem: by combining conforming and nonconforming finite element methods, Sci. China Math., 55 (2012), pp. 1069-1082.

[28] M. S. Min AND D. GotTLieb, Domain decomposition spectral approximations for an eigenvalue problem with a piecewise constant coefficient, SIAM J. Numer. Anal., 43 (2005), pp. 502-520.

[29] L. MU, J. WANG, Y. WANG, AND X. YE, A computational study of the weak Galerkin method for second-order elliptic equations, Numer. Algorithms, 63 (2013), pp. 753-777.

[30] L. MU, J. WANG, AND X. YE, A weak Galerkin finite element method with polynomial reduction, J. Comput. Appl. Math., 285 (2015), pp. 45-58.

[31] L. MU, J. WANG, X. YE, AND S. ZHANG, A C0-weak Galerkin finite element method for the biharmonic equation, J. Sci. Comput., 59 (2014), pp. 473-495.

[32] L. MU, J. WANG, X. YE, AND S. ZHAO, A Numerical Study on the Weak Galerkin Method for the Helmholtz Equation, Commun. Comput. Phys., 15 (2014), pp. 1461-1479.

[33] M. PLUM, Explicit H2-estimates and pointwise bounds for solutions of second-order elliptic boundary value problems, J. Math. Anal. Appl., 165 (1992), pp. 36-61.

[34] I. $v$ SEBESTOVÁ AND T. VEJCHODSKÝ, Two-sided bounds for eigenvalues of differential operators with applications to Friedrichs, Poincaré, trace, and similar constants, SIAM J. Numer. Anal., 52 (2014), pp. 308-329.

[35] G. Strang, G. J. Fix, AND D. S. GRIFrin, An Analysis of the Finite-Element Method, J. Appl. Mech., 41 (1974), p. 62. 
[36] J. WANG, R. WANG, Q. ZHAI, AND R. ZHANG, A Systematic Study on Weak Galerkin Finite Element Methods for Second Order Elliptic Problems, J. Sci. Comput., (2017), pp. 1-28.

[37] J. WANG AND X. YE, A weak Galerkin finite element method for second-order elliptic problems, J. Comput. Appl. Math., 241 (2013), pp. 103-115.

[38] _ A weak Galerkin mixed finite element method for second order elliptic problems, Math. Comput., 83 (2014), pp. 2101-2126.

[39] J. WANG, X. YE, Q. ZHAI, AND R. ZHANG, Discrete maximum principle for the P1 P0 weak Galerkin finite element approximations, J. Comput. Phys., 362 (2018), pp. 114-130.

[40] Q. ZHAI, X. HU, AND R. A. N. ZHANG, The shifted-inverse power weak galerkin method for eigenvalue problems, arXiv:1803.09192, pp. 1-19.

[41] Q. ZHAi, H. XIE, R. ZHANG, AND Z. ZHANG, Acceleration of weak Galerkin methods for the Laplacian eigenvalue problem, arXiv:1708.08183, pp. 1-20.

[42] Q. ZHAI AND R. ZHANG, Lower and upper bounds of Laplacian eigenvalue problem by weak Galerkin method on triangular meshes, Discret. Contin. Dyn. Syst. - B, Accepted (2018).

[43] Q. Zhai, R. Zhang, N. MAlluwaWAdu, AND S. Hussain, The weak Galerkin method for linear hyperbolic equation, Commun. Comput. Phys., 24 (2018), pp. 152-166.

[44] Q. ZHAI, R. ZHANG, AND L. MU, A new weak Galerkin finite element scheme for the Brinkman model, Commun. Comput. Phys., 19 (2016), pp. 1409-1434.

[45] Q. ZHAI, R. ZHANG, AND X. WANG, A hybridized weak Galerkin finite element scheme for the Stokes equations, Sci. China Math., 58 (2015), pp. 2455-2472.

[46] J. ZhanG, K. ZhANG, J. LI, AND X. WANG, A Weak Galerkin Finite Element Method for the Navier-Stokes Equations, Commun. Comput. Phys., 23 (2018), pp. 706-746.

[47] Q. ZHANG AND R. ZHANG, A weak Galerkin mixed finite element method for second-order elliptic equations with Robin boundary conditions, J. Comput. Math., 34 (2016), pp. 532-548.

[48] R. ZHANG AND Q. ZHAI, A weak Galerkin finite element scheme for the biharmonic equations by using polynomials of reduced order, J. Sci. Comput., 64 (2015), pp. 559-585.

[49] X. ZHENG AND X. XIE, A posteriori error estimator for a weak Galerkin finite element solution of the Stokes problem, East Asian J. Applied Math., 7 (2017), pp. 508-529. 